\title{
Unsaturation Elements and other Modifications of Phospholipids in Bacteria: New Insight from UVPD Mass Spectrometry
}

Molly S. Blevins ${ }^{1}$, Virginia K. James ${ }^{1}$, Carmen M. Herrera², Alexandria B. Purcell ${ }^{2}$, M. Stephen Trent ${ }^{2,3,4}$ and Jennifer S. Brodbelt ${ }^{1^{*}}$

${ }^{1}$ Department of Chemistry, University of Texas at Austin, Austin, TX 78712

${ }^{2}$ Department of Infectious Diseases, College of Veterinary Medicine, University of Georgia, Athens, GA 30602

${ }^{3}$ Department of Microbiology, College of Arts and Sciences, University of Georgia, Athens, GA 30602

${ }^{4}$ Center for Vaccines and Immunology, University of Georgia, Athens, GA 30602

*Corresponding author: jbrodbelt@cm.utexas.edu

\section{Supporting Information}

$\underline{\text { Table of Contents }}$

\begin{tabular}{|c|c|}
\hline Content & $\begin{array}{c}\text { Page } \\
\text { no. }\end{array}$ \\
\hline Fig S1. Base peak LC-MS traces of bacterial lipid extracts & S2 \\
\hline Fig S2. Omega vs. delta nomenclature for classification of GPL unsaturation elements & S3 \\
\hline Fig S3. Chromatographic separation of hydroxyl lipids and their non-hydroxyl isobaric counterparts & S4 \\
\hline Fig S4. Possible headgroup structures for Me-PS headgroup & S5 \\
\hline Fig S5. P. aeruginosa lipid extract base peak LC-MS trace with XIC of Me-PS lipid & S6 \\
\hline Fig S6. C. jejuni lipid extract base peak LC-MS trace with XIC of lysyl-PG lipid & S7 \\
\hline Table S1. List of investigated bacterial lipid extracts & S8 \\
\hline Table S2. List of identified unsaturated lipids in E. coli lipid extract with $\mathrm{m} / \mathrm{z}$ and $\mathrm{ppm}$ error values & S9 \\
\hline Table S3. List of identified unsaturated lipids in E. cloacae lipid extract with $\mathrm{m} / \mathrm{z}$ and $\mathrm{ppm}$ error values & S10 \\
\hline Table S4. List of identified unsaturated lipids in $K$. pneumoniae lipid extract with $\mathrm{m} / \mathrm{z}$ and ppm error values & S11 \\
\hline Table S5. List of identified unsaturated lipids in $P$. mirabilis lipid extract with $\mathrm{m} / \mathrm{z}$ and $\mathrm{ppm}$ error values & S12 \\
\hline Table S6. List of identified unsaturated lipids in $P$. aeruginosa lipid extract with $\mathrm{m} / \mathrm{z}$ and ppm error values & S13 \\
\hline Table S7. List of identified unsaturated lipids in S. typhimurium lipid extract with $\mathrm{m} / \mathrm{z}$ and ppm error values & S14 \\
\hline Table S8. List of identified unsaturated lipids in $V$. cholerae lipid extract with $\mathrm{m} / \mathrm{z}$ and $\mathrm{ppm}$ error values & S15 \\
\hline Table S9. List of identified unsaturated lipids in S. marcescens lipid extract with $\mathrm{m} / \mathrm{z}$ and ppm error values & S16 \\
\hline Table S10. List of identified unsaturated lipids in C. jejuni lipid extract with $\mathrm{m} / \mathrm{z}$ and ppm error values & S17 \\
\hline $\begin{array}{l}\text { Table S11. List of identified unsaturated lipids in Y. pseudotuberculosis lipid extract with } \mathrm{m} / \mathrm{z} \text { and ppm error } \\
\text { values }\end{array}$ & S18 \\
\hline Table S12. List of identified unsaturated lipids in B. subtilis lipid extract with $\mathrm{m} / \mathrm{z}$ and $\mathrm{ppm}$ error values & S19 \\
\hline Table S13. List of identified unsaturated lipids in L. plantarum lipid extract with $\mathrm{m} / \mathrm{z}$ and ppm error values & S20 \\
\hline Table S14. List of identified PUFAs within GPLs with $\mathrm{m} / \mathrm{z}$ and $\mathrm{ppm}$ error values & S21 \\
\hline Table S15. List of identified hydroxyl acyl chains within GPLs $m / z$ and $p p m$ error values & S22 \\
\hline $\begin{array}{l}\text { Table S16. List of identified } \mathrm{OH} \text { acyl chains with acyl chain product ion } m / z \text { values and } m / z \text { values of isobaric } \\
\text { unmodified (non-hydroxyl) acyl chains }\end{array}$ & S23 \\
\hline Table S17. List of lysyl-PGs in Gram-negative C. jejuni and Gram-positive B. subtilis and L. plantarum & S24 \\
\hline
\end{tabular}



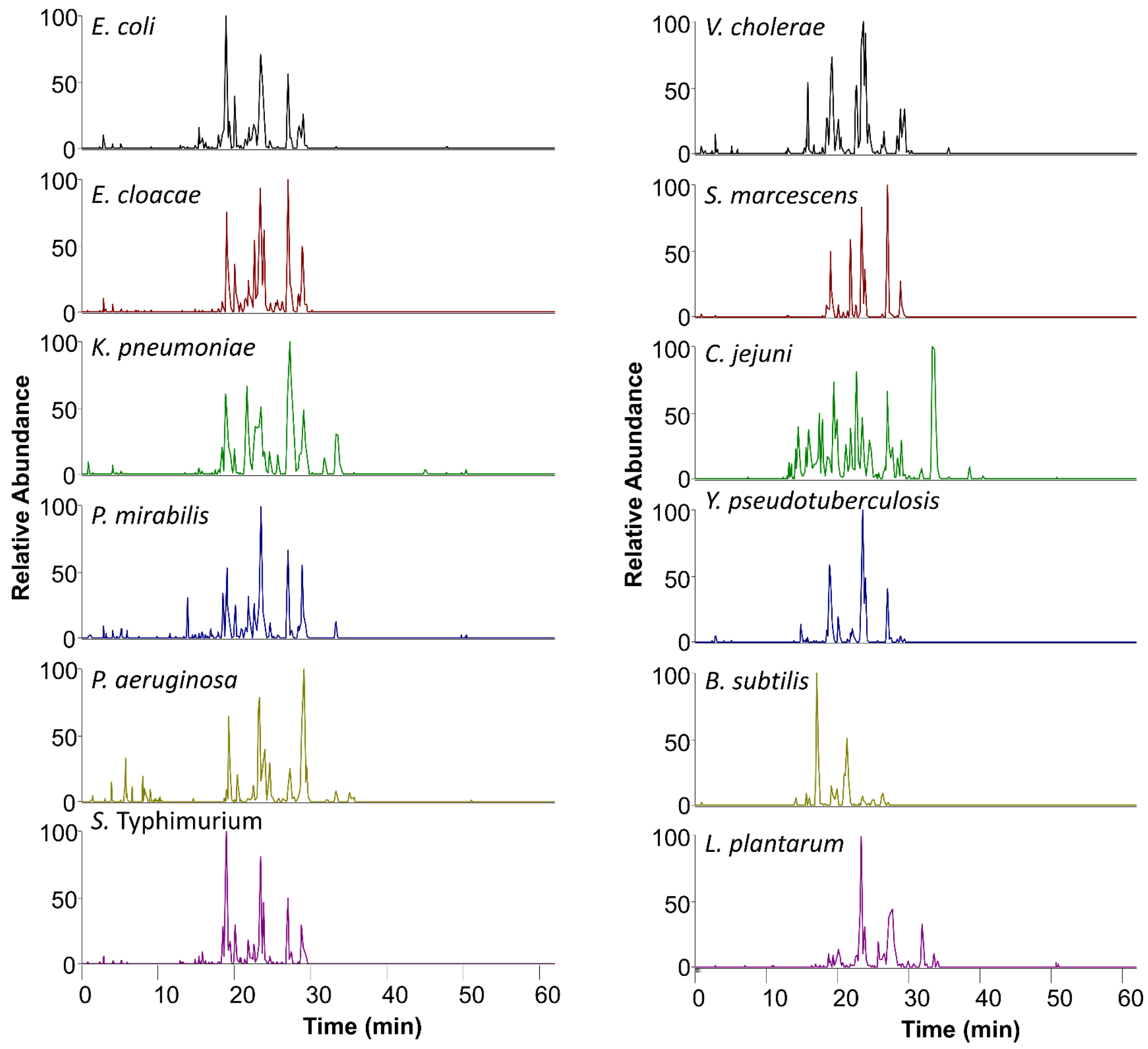

Figure S1. Base peak LC-MS traces of all 12 investigated bacterial lipid extracts. 


\section{PG 16:0/16:1(9Z)}

\section{PG 16:0/16:1(cis $\omega-7)$}<smiles>CCCCCCCCCCCCCCCCCCCC(=O)OC[C@H](C)COP(=O)([O-])OC[C@H](O)CO</smiles>

\section{PG 16:0/18:1(11Z)}

\section{PG 16:0/18:1(cis $\omega-7)$}

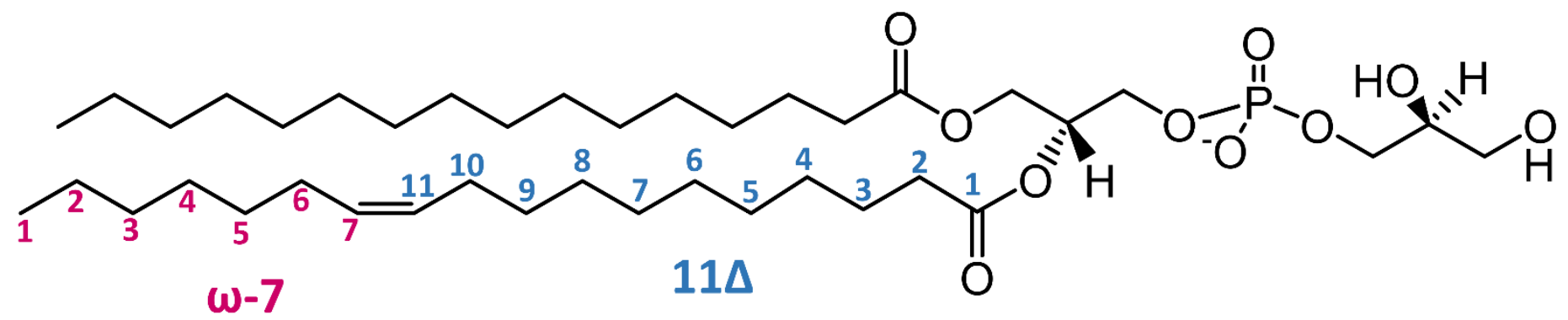

Figure S2. The omega nomenclature ( $\omega-7)$ in pink font allows simple recognition of unsaturation elements which are found at the same position from the terminal acyl chain carbon end despite having different numbers of carbon atoms within the acyl chains. The contrasting delta nomenclature is shown in blue font. 


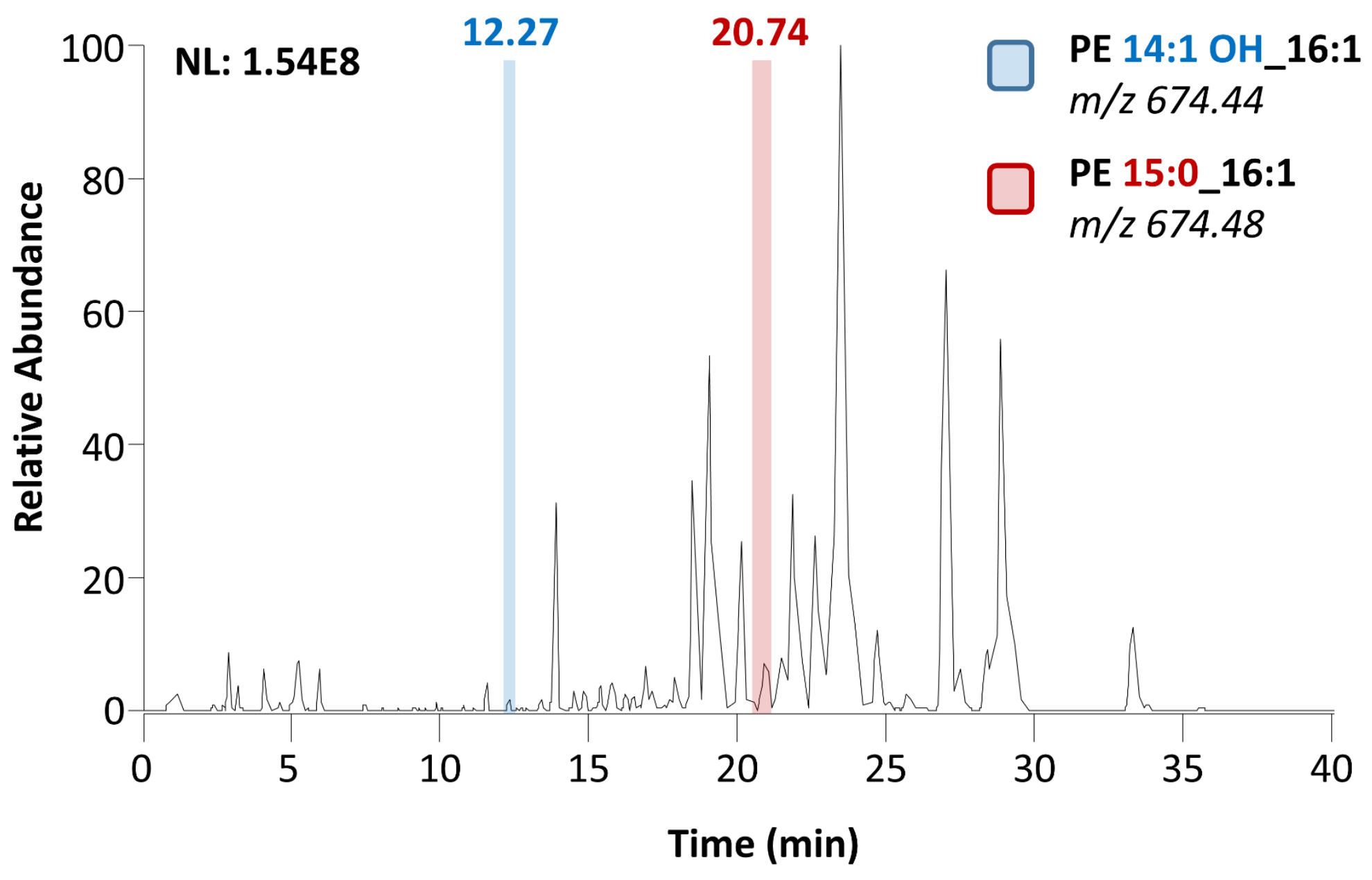

Figure S3. Example of marked differences in retention time of two lipids - one containing an unsaturated hydroxyl acyl chain (PE 14:1 OH_16:1 of $\mathrm{m} / \mathrm{z}$ 674.44) and its non-hydroxyl lipid counterpart (PE 15:0_16:1 of $\mathrm{m} / \mathrm{z}$ 674.48) from $P$. mirabilis lipid extract. With lack of high-resolution instrumentation, differences in retention time enable differentiation (albeit not complete characterization) of hydroxyl lipids and their isobaric non-hydroxyl counterparts with a mass difference of $0.04 \mathrm{Da}$. 

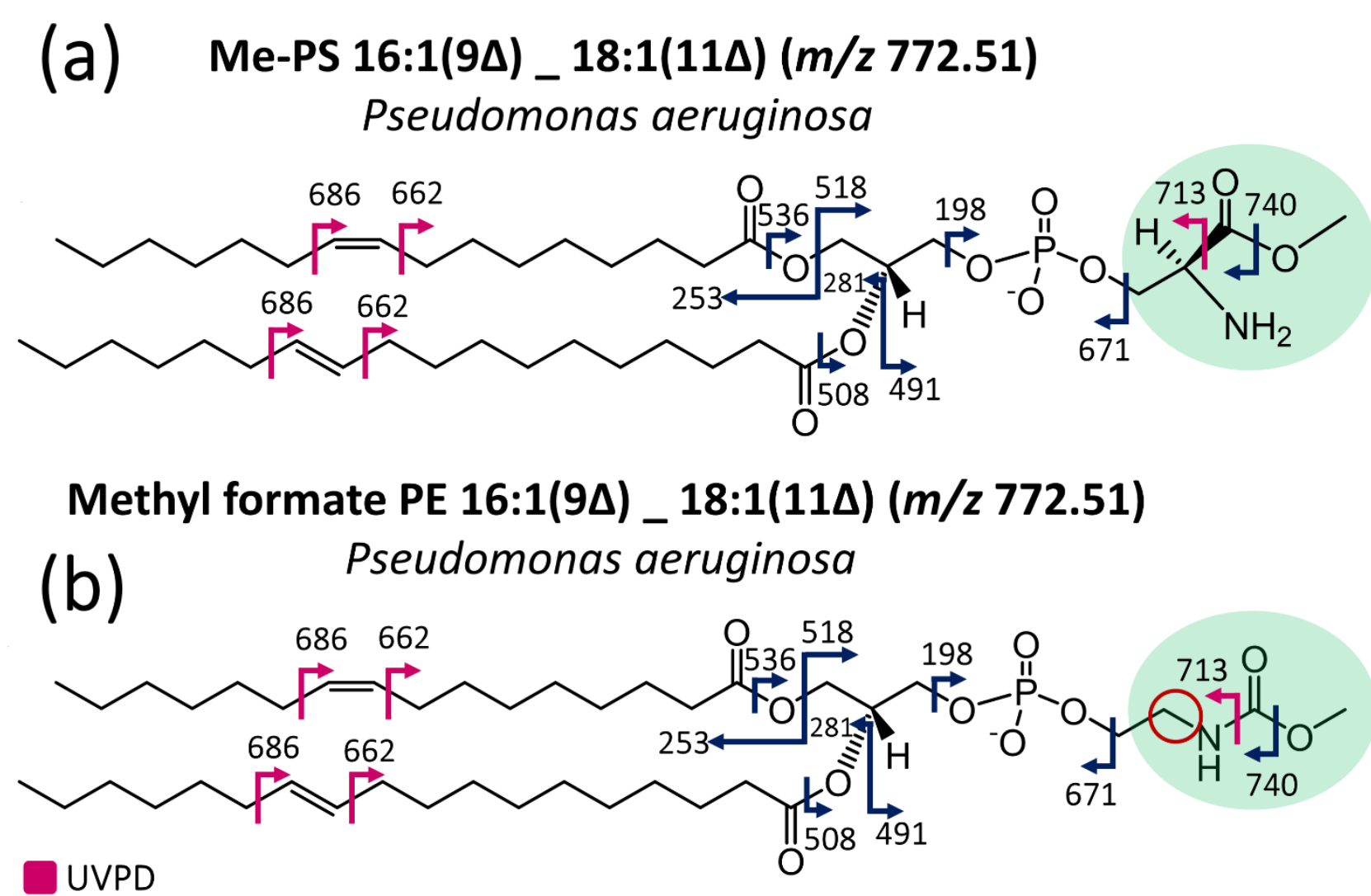

HCD \& UVPD

Figure S4. Two possible structures for the observed unique headgroup as shown for a lipid of $\mathrm{m} / \mathrm{z}$ 772.51 from $P$. aeruginosa lipid extract; (a) Me-PS type lipid and (b) modified PE headgroup with methyl formate. Both structural possibilities align with the measured intact mass and fragmentation patterns. Hypothetical cleavage of the C-N bond circled in red in (b) (theoretical $\mathrm{m} / \mathrm{z} 698$ ) could unambiguously identify the correct structure for this headgroup, but is not observed, making (a) the more likely structure. 


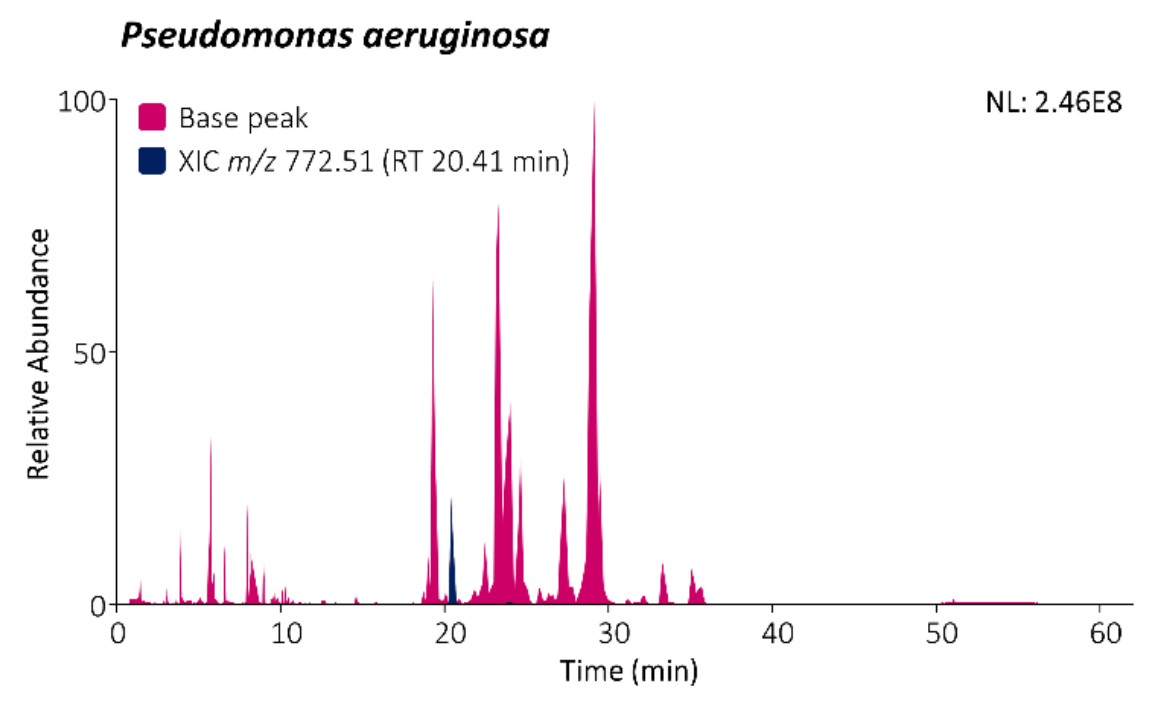

Figure S5. Base peak LC-MS trace of Pseudomonas aeruginosa lipid extract with XIC of $\mathrm{m} / \mathrm{z} 772.51$

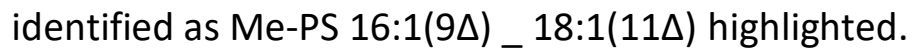




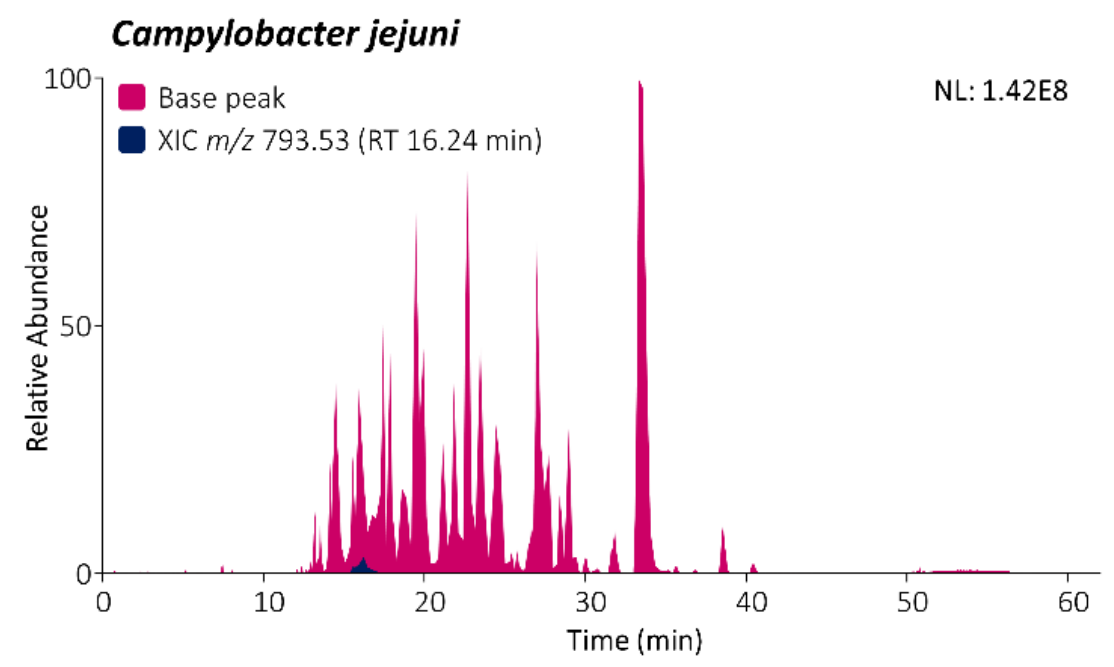

Figure S6. Base peak LC-MS trace of Campylobacter jejuni lipid extract with XIC of $m / z 793.53$ identified as Lysyl-PG 13:0_15:0 highlighted. 


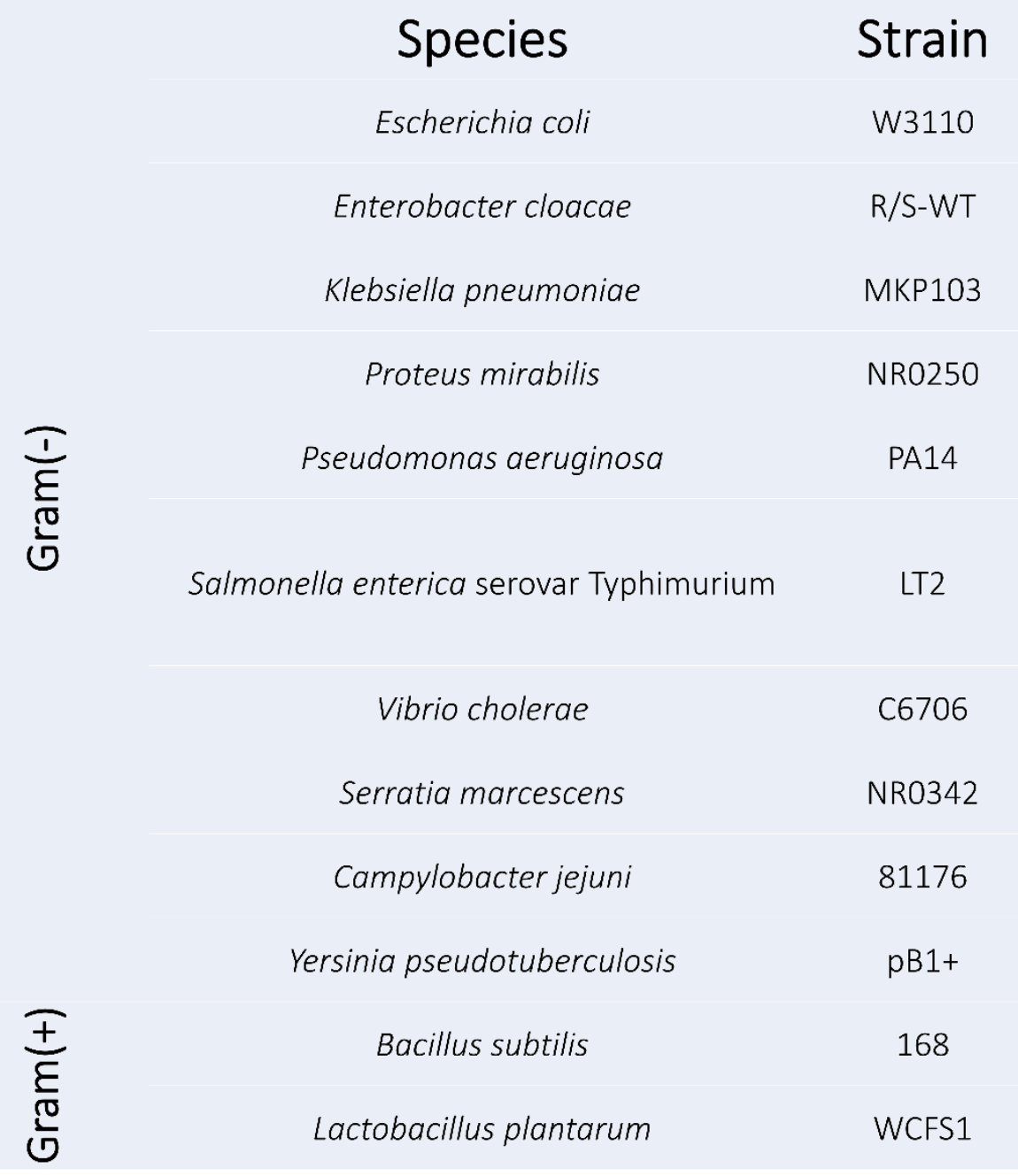

Table S1. List of investigated bacterial lipid extracts including $10 \mathrm{Gram}$-negative and $2 \mathrm{Gram}$-positive species. 


\section{E. coli}

\begin{tabular}{|c|c|c|c|c|c|c|c|c|c|c|c|c|c|c|c|c|c|c|c|c|}
\hline $\begin{array}{l}\text { Precurso } \\
\mathrm{r} \mathrm{m} / \mathrm{z}\end{array}$ & $\begin{array}{l}\text { Precursor } \mathrm{m} / \mathrm{z} \\
\text { (theo) }\end{array}$ & $\begin{array}{l}\text { ppm } \\
\text { error }\end{array}$ & $\mathrm{RT}$ (min) & $\begin{array}{c}\text { Headgro } \\
\text { up }\end{array}$ & $\begin{array}{c}\text { acyl chain } \\
1\end{array}$ & $\mathrm{~m} / \mathrm{z} \quad \begin{array}{c}\mathrm{m} / \mathrm{z} \\
\text { (theo) }\end{array}$ & $\begin{array}{l}\text { ppm } \\
\text { error }\end{array}$ & $\begin{array}{c}\text { acyl chain } \\
2\end{array}$ & $n \mathrm{~m} / \mathrm{z}$ & $\begin{array}{c}\mathrm{m} / \mathrm{z} \\
\text { (theo) }\end{array}$ & $\begin{array}{l}\text { ppm } \\
\text { error }\end{array}$ & $\begin{array}{c}\text { Unsat lon } \\
1 \mathrm{~m} / \mathrm{z}\end{array}$ & $\begin{array}{l}\text { Unsat lon } \\
1 \mathrm{~m} / \mathrm{z} \\
\text { (theo) }\end{array}$ & $\begin{array}{l}\text { Unsat Ion } 1 \\
\text { ppm error }\end{array}$ & $\begin{array}{l}\text { Unsat lon } \\
2 \mathrm{~m} / \mathrm{z}\end{array}$ & $\begin{array}{l}\text { Unsat lon } \\
2 \mathrm{~m} / \mathrm{z} \\
\text { (theo) }\end{array}$ & $\begin{array}{l}\text { Unsat lon } 2 \\
\text { ppm error }\end{array}$ & $\begin{array}{l}\text { Differen } \\
\text { ce (Da) }\end{array}$ & $\begin{array}{c}\text { Unsat } \\
\text { Position } \\
\text { (Delta) }\end{array}$ & $\begin{array}{l}\text { Unsat Position } \\
\text { (Omega) }\end{array}$ \\
\hline 691.4562 & 691.4556 & 0.9 & 15.48 & PG & $14: 0$ & 227.2018227 .2017 & 0.4 & $16: 1$ & 253.2175 & 253.2173 & 0.8 & 581.3485 & 581.346 & 4.3 & 605.3403 & 605.346 & -9.4 & 23.99 & $9 \Delta$ & $\omega-7$ \\
\hline 646.4453 & 646.4453 & 0.0 & 17.02 & $\mathrm{PE}$ & $12: 0$ & 199.1703199 .1704 & -0.5 & 17:1 & 267.2329 & 267.2330 & -0.4 & 534.3184 & 534.3201 & -3.2 & 548.3322 & 548.3358 & -6.6 & 14.01 & $9 \Delta$ & $\omega-7$ \\
\hline 705.4713 & 705.4712 & 0.1 & 17.56 & PG & $14: 0$ & 227.2017227 .2017 & 0.0 & $17: 1$ & 267.2330 & 267.2330 & 0.0 & 593.3423 & 593.346 & -6.2 & 607.3586 & 607.3617 & -5.1 & 14.02 & $9 \Delta$ & $\omega-7$ \\
\hline 674.4738 & & -4.2 & 20.86 & & 15:0 & 241.2176241 .2173 & 1.2 & $16: 1$ & 253.2176 & 253.2173 & 1.2 & 564.3688 & 564.3671 & 3.0 & 588.3705 & 588.3671 & 5.8 & 24.00 & & \\
\hline 674.4781 & & 2.2 & 21.33 & & $14: 0$ & 227.202227 .2017 & 1.3 & 17:1 & 267.2334 & 267.2330 & 1.5 & 562.3510 & 562.3514 & -0.7 & 576.3656 & 576.3671 & -2.6 & 14.01 & & \\
\hline 733.504 & 733.5025 & 2.0 & 21.63 & PG & $16: 0$ & 255.2329255 .2330 & -0.4 & $17: 1$ & 267.2328 & 267.2330 & 0.7 & 621.3748 & 621.3773 & 4.0 & 635.3902 & 635.393 & -4.4 & 14.02 & $9 \Delta$ & $\omega-7$ \\
\hline 688.4968 & & 6.5 & 23.29 & $\mathrm{PE}$ & $16: 1$ & 253.219253 .2173 & 6.7 & $16: 0$ & 255.2333 & 255.2330 & 1.2 & 578.3784 & 578.3827 & -7.4 & 602.382 & 602.3827 & -1.2 & 24.00 & $9 \Delta$ & $\omega-7$ \\
\hline 688.4935 & & 1.7 & 24.15 & $P E$ & $15: 0$ & 241.2176241 .2173 & 1.2 & $17: 1$ & 267.2332 & 267.2330 & 0.7 & 576.3669 & 576.3671 & -0.3 & 590.3782 & 590.3827 & -7.6 & 14.01 & $9 \Delta$ & $\omega-7$ \\
\hline 773.5356 & 773.5338 & 2.3 & 24.00 & PG & $18: 1$ & 281.2488281 .2486 & 0.7 & $18: 1$ & 281.2488 & 281.2486 & 0.7 & 663.4229 & 663.4243 & -2.1 & 687.4188 & 687.4243 & -8.0 & 24.00 & $11 \Delta$ & $\omega-7$ \\
\hline 714.5084 & 714.5079 & 0.7 & 24.59 & $\mathrm{PE}$ & $17: 1$ & 267.2329267 .2330 & -0.4 & $17: 1$ & 267.2329 & 267.2330 & -0.4 & 602.3824 & 602.3827 & -0.5 & 616.3964 & 616.3984 & -3.2 & 14.01 & $9 \Delta$ & $\omega-7$ \\
\hline 702.5106 & 702.5079 & 3.8 & 26.82 & $\mathrm{PE}$ & $16: 0$ & 255.2335255 .2330 & 2.0 & $17: 1$ & 267.2336 & 267.2330 & 2.2 & 590,3806 & 590.3827 & -3.6 & 604.3992 & 604.3984 & 1.3 & 14.02 & $9 \Delta$ & $\omega-7$ \\
\hline 716.5267 & 716.5236 & 4.3 & 29.02 & $\mathrm{PE}$ & $16: 0$ & 255.2337255 .2330 & 2.7 & 18:1 & 281.2495 & 281.2486 & 3.2 & 606.418 & 606.414 & 6.6 & 630.4118 & 630.414 & -3.5 & 23.99 & $11 \Delta$ & $\omega-7$ \\
\hline 742.5407 & 742.5392 & 2.0 & 29.47 & $\mathrm{PE}$ & $18: 1$ & 281.2491281 .2486 & 1.8 & $18: 1$ & 281.2491 & 281.2486 & 1.8 & 632.4254 & 632.4297 & 6.8 & 656.429 & 656.4297 & -1.1 & 24.00 & $11 \Delta$ & $\omega .7$ \\
\hline 730.5395 & & 0.4 & 33.15 & & $16: 0$ & 255.233255 .2330 & 0.0 & 19:1 & 295.2643 & 295.2639 & 1.4 & 618.4097 & & -7.0 & 632.4281 & & -2.5 & 14.02 & $11 \Delta$ & $\omega-7$ \\
\hline 730.5394 & & 0.3 & 33.62 & & 17:1 & 267.2329267 .233 & -0.4 & 18:0 & 283.2643 & 283.2643 & 0.0 & 618.4081 & & -9.5 & 632.426 & & -5.9 & 14.02 & $9 \Delta$ & $\omega-7$ \\
\hline
\end{tabular}

Table S2. List of all identified unsaturated lipids in E. coli lipid extract with all precursor and fragment ion ppm error values for acyl chain product ions and unsaturation element fragment ions (within 10 ppm error). Features are color coded by headgroup (PGs in red, PEs in yellow, PCs in purple), unsaturation type (cyclopropane rings in blue, double bonds in green), and unique acyl chain modifications (odd-carbon branched acyl chains in pink, hydroxyl acyl chains in brown). 


\section{E. cloacae}

\begin{tabular}{|c|c|c|c|c|c|c|c|c|c|c|c|c|c|c|c|c|c|c|c|c|}
\hline $\begin{array}{l}\text { Precurso } \\
r \mathrm{~m} / \mathbf{z}\end{array}$ & $\begin{array}{l}\text { Precursor } \mathrm{m} / \mathrm{z} \\
\text { (theo) }\end{array}$ & $\begin{array}{l}\text { ppm } \\
\text { error }\end{array}$ & $\mathrm{RT}(\min )$ & $\begin{array}{l}\text { Headgro } \\
\text { up }\end{array}$ & $\begin{array}{c}\text { acyl chain } \\
1\end{array}$ & $\mathrm{~m} / \mathrm{z}^{\mathrm{m}} \begin{array}{c}\mathrm{m} / \mathrm{z} \\
\text { (theo) }\end{array}$ & $\begin{array}{l}\text { ppm } \\
\text { error }\end{array}$ & $\begin{array}{c}\text { acyl chain } \\
2\end{array}$ & $\mathrm{~m} / \mathrm{z}$ & $\begin{array}{c}\mathrm{m} / \mathrm{z} \\
\text { (theo) }\end{array}$ & $\begin{array}{l}\text { ppm } \\
\text { error }\end{array}$ & $\begin{array}{l}\text { Unsat lon } \\
1 \mathrm{~m} / \mathrm{z}\end{array}$ & $\begin{array}{c}\text { Unsat lon } \\
1 \mathrm{~m} / \mathrm{z} \\
\text { (theo) }\end{array}$ & $\begin{array}{l}\text { Unsat lon } 1 \\
\text { ppm error }\end{array}$ & $\begin{array}{l}\text { Unsat lon } \\
2 \mathrm{~m} / \mathrm{z}\end{array}$ & $\begin{array}{c}\text { Unsat lon } \\
2 \mathrm{~m} / \mathrm{z} \\
\text { (theo) }\end{array}$ & $\begin{array}{l}\text { Unsat lon } 2 \\
\text { ppm error }\end{array}$ & $\begin{array}{l}\text { Differen } \\
\text { ce (Da) }\end{array}$ & $\begin{array}{c}\text { Unsat } \\
\text { Position } \\
\text { (Delta) }\end{array}$ & $\begin{array}{c}\text { Unsat } \\
\text { Position } \\
\text { (Omega) }\end{array}$ \\
\hline 632.4302 & 632.4297 & 0.8 & 14.79 & PE & 12:0 & 199.1704199 .1704 & 0.0 & $16: 1$ & 253.2174 & 253.2173 & 0.4 & 522.3250 & 522.3201 & 9.4 & 546.3161 & 546.3201 & .7 .3 & 23.99 & $9 \Delta$ & $\omega-7$ \\
\hline 691.4559 & 691.4556 & 0.4 & 15.33 & PG & 14:0 & 227.2018227 .2017 & 0.4 & $16: 1$ & 253.2174 & 253.2173 & 0.4 & 581.3483 & 581.346 & 4.0 & 605.3417 & 605.346 & -7.1 & 23.99 & $9 \Delta$ & $\omega-7$ \\
\hline 646.4458 & 646.4453 & 0.8 & 17.11 & PE & 12:0 & 199.1705199 .1704 & 0.5 & 17:1 & 267.2331 & 267.2330 & 0.4 & 534.319 & 534.3201 & -2.1 & 548.3337 & 548.3358 & -3.8 & 14.01 & $9 \Delta$ & $\omega-7$ \\
\hline 705.4714 & 705.4712 & 0.3 & 17.57 & PG & 14:0 & 227.2017227 .2017 & 0.0 & 17:1 & 267.2330 & 267.2330 & 0.0 & 593.3426 & 593.346 & -5.7 & 607.3588 & 607.3617 & 4.8 & 14.02 & $9 \Delta$ & $\omega 7$ \\
\hline 719.4877 & 719.4869 & 1.1 & 19.09 & PG & $16: 0$ & 255.2329 255.2330 & -0.4 & $16: 1$ & 253.2176 & 253.2173 & 1.2 & 609.3812 & 609.3773 & 6.4 & 633.3773 & 633.3773 & 0.0 & 24.00 & $9 \Delta$ & $\omega-7$ \\
\hline 686.4773 & 686.4766 & 1.0 & 19.25 & PE & $16: 1$ & 253.2174253 .2173 & 0.4 & $16: 1$ & 253.2174 & 253.2173 & 0.4 & 576.3707 & 576.3671 & 6.2 & 600.3616 & 600.3671 & -9.2 & 23.99 & $9 \Delta$ & $\omega-7$ \\
\hline 674.4785 & & 2.8 & 20.82 & & 15:0 & 241.2177241 .2173 & 1.7 & $16: 1$ & 253.2178 & 253.2173 & 2.0 & 564.3664 & 564.3671 & 1.2 & 588.3699 & 588.3671 & 4.8 & 24.00 & $9 \Delta$ & $\omega 7$ \\
\hline 674.4791 & & 3.7 & 21.37 & & 14:0 & 227.2022227 .2017 & 2.2 & $17: 1$ & 267.2336 & 267.2330 & 2.2 & 562.3516 & 562.3514 & 0.4 & 576.366 & 576.3671 & -1.9 & 14.01 & $9 \Delta$ & $\omega-7$ \\
\hline 733.5064 & 733.5025 & 5.3 & 21.88 & PG & $16: 0$ & 255.2338 255.2330 & 3.1 & 17:1 & 267.2339 & 267.2330 & 3.4 & 621.3762 & 621.3773 & -1.8 & 635.3918 & 635.393 & -1.9 & 14.02 & $9 \Delta$ & $w-7$ \\
\hline 688.4983 & & 8.7 & 23.39 & & 16:1 & 253.2195253 .2173 & 8.7 & $16: 0$ & 255.2334 & 255.2330 & 1.6 & 578.379 & 578.3827 & 6.4 & 602.3827 & 602.3827 & 0.0 & 24.00 & $9 \Delta$ & $\omega 7$ \\
\hline 688.4945 & & 3.2 & 24.21 & & $15: 0$ & 241.2178241 .2173 & 2.1 & $17: 1$ & 267.2336 & 267.2330 & 2.2 & 576.3666 & 576.3671 & -0.9 & 590.3792 & 590.3827 & -5.9 & 14.01 & $9 \Delta$ & $\omega-7$ \\
\hline \multirow{2}{*}{714.5082} & \multirow{2}{*}{714.5079} & \multirow{2}{*}{0.4} & \multirow{2}{*}{24.66} & \multirow{2}{*}{ PE } & 16:1 & 253.2174253 .2173 & 0.4 & $18: 1$ & 281.2502 & 281.2486 & 5.7 & 604.3972 & 604.3984 & -2.0 & 628.3955 & 628.3984 & -4.6 & 24.00 & $\begin{array}{l}9 \Delta(16: 1), \\
11 \Delta(18: 1)\end{array}$ & $\omega-7, \omega-7$ \\
\hline & & & & & 17:1 & 267.2331267 .2330 & 0.4 & $17: 1$ & 267.2331 & 267.2330 & 0.4 & 602.3776 & 602.3827 & -8.5 & 616.3969 & 616.3984 & -2.4 & 14.02 & $9 \Delta, 9 \Delta$ & $\omega-7, \omega-7$ \\
\hline 702.5103 & \multirow{2}{*}{702.5079} & 3.4 & 26.28 & \multirow{2}{*}{ PE } & 16:1 & 253.2178253 .2173 & 2.0 & $17: 0$ & 269.2492 & 269.2486 & 2.2 & 592.3999 & 592.3984 & 2.5 & 616.3953 & 616.3984 & -5.0 & 24.00 & $9 \Delta$ & $\omega-7$ \\
\hline 702.5106 & & 3.8 & 26.81 & & $16: 0$ & 255.2335255 .2330 & 2.0 & $17: 1$ & 267.2336 & 267.2330 & 2.2 & 590.3806 & 590.3827 & -3.6 & 604.3992 & 604.3984 & 1.3 & 14.02 & $9 \Delta$ & $\omega-7$ \\
\hline 728.5271 & 728.5236 & 4.8 & 27.49 & $P E$ & 17:1 & 267.2337267 .2330 & 2.6 & 18:1 & 281.2495 & 281.2486 & 3.2 & & & -5.2 & 642.4083 & & 8.9 & 24.00 & $\begin{array}{l}9 \Delta \Delta(17: 1), \\
11 \Delta(18: 1)\end{array}$ & $\omega-7$ \\
\hline 716.5265 & & 4.0 & 28.86 & & 16:0 & 255.2337255 .2330 & 2.7 & $18: 1$ & 281.2494 & 281.2486 & 2.8 & 606.4182 & 606.114 & 6.9 & 630.412 & 630.414 & -3.2 & 23.99 & $11 \Delta$ & $\omega-7$ \\
\hline 716.5243 & & 1.0 & 30.14 & & 17:1 & 267.2332267 .2330 & 0.7 & $17: 0$ & 269.2486 & 269.2486 & 0.0 & 604.3951 & 604.3984 & -5.5 & 618.412 & 618.414 & -3.2 & 14.02 & $9 \Delta$ & $\omega-7$ \\
\hline \multirow[t]{2}{*}{730.5397} & \multirow[t]{2}{*}{730.5392} & \multirow[t]{2}{*}{0.7} & \multirow[t]{2}{*}{33.48} & \multirow[t]{2}{*}{ PE } & 16:0 & 255.233255 .2330 & 0.0 & 19:1 & 295.2643 & 295.2639 & 1.4 & \multirow[t]{2}{*}{618.41} & \multirow[t]{2}{*}{618.414} & -6.5 & 632.4272 & 632.4297 & -4.0 & 14.02 & $\begin{array}{l}9 \Delta(17: 1), \\
11 \Delta(19: 1)\end{array}$ & $\omega-7, \omega-7$ \\
\hline & & & & & 17:1 & 267.2330267 .2330 & 0.0 & 18:0 & 283.2644 & 283.2643 & 0.4 & & & & & & & & & \\
\hline
\end{tabular}

Table S3. List of all identified unsaturated lipids in E. cloacae lipid extract with all precursor and fragment ion ppm error values for acyl chain product ions and unsaturation element fragment ions (within 10 ppm error). Features are color coded by headgroup (PGs in red, PEs in yellow, PCs in purple), unsaturation type (cyclopropane rings in blue, double bonds in green), and unique acyl chain modifications (odd-carbon branched acyl chains in pink, hydroxyl acyl chains in brown). 


\section{K. pneumoniae}

\begin{tabular}{|c|c|c|c|c|c|c|c|c|c|c|c|c|c|c|c|c|c|c|c|c|}
\hline $\begin{array}{l}\text { Precursor } \\
\mathrm{m} / \mathrm{z}\end{array}$ & $\begin{array}{l}\text { Precursor } \mathrm{m} / \mathrm{z} \\
\text { (theo) }\end{array}$ & $\begin{array}{l}\text { ppm } \\
\text { error }\end{array}$ & $\mathrm{RT}(\mathrm{min})$ & $\begin{array}{c}\text { Headgro } \\
\text { up }\end{array}$ & $\begin{array}{c}\text { acyl chain } \\
1\end{array}$ & $n=\begin{array}{c}m / 2 \\
m / 2 \\
\text { (theo) }\end{array}$ & $\begin{array}{l}\mathrm{ppm} \\
\text { error }\end{array}$ & $\begin{array}{c}\text { acyl chain } \\
2\end{array}$ & $\mathrm{~m} / \mathrm{z}$ & $\begin{array}{c}\mathrm{m} / \mathrm{z} \\
\text { (theo) }\end{array}$ & $\begin{array}{l}\text { ppm } \\
\text { error }\end{array}$ & $\begin{array}{l}\text { Unsat lon } \\
1 \mathrm{~m} / 2\end{array}$ & $\begin{array}{l}\text { Unsat lon } \\
1 \mathrm{~m} / \mathrm{z} \\
\text { (theo) }\end{array}$ & $\begin{array}{l}\text { Unsat lon } 1 \\
\text { ppm error }\end{array}$ & $\begin{array}{l}\text { Unsat lon } \\
2 \mathrm{~m} / 2\end{array}$ & $\begin{array}{c}\text { Unsat lon } \\
2 \mathrm{~m} / 2 \\
\text { (theo) }\end{array}$ & $\begin{array}{l}\text { Unsat lon } 2 \\
\text { ppm error }\end{array}$ & $\begin{array}{c}\text { Differenc } \\
\text { e (Da) }\end{array}$ & $\begin{array}{l}\text { Unsat } \\
\text { Position } \\
\text { (Delta) }\end{array}$ & $\begin{array}{l}\text { Unsat Position } \\
\text { (Omega) }\end{array}$ \\
\hline 717.4713 & 717.4712 & 0.1 & 15.72 & PG & 16:1 & 253.2173253 .2173 & 0.0 & $16: 1$ & 253.2173 & 253.2173 & 0.0 & 607.3639 & 607.3617 & 3.6 & 631.3564 & 631.3617 & -8.4 & 23.99 & $9 \Delta$ & $\omega-7$ \\
\hline 646.4460 & 646.4453 & 1.1 & 17.06 & $\mathrm{PE}$ & 12:0 & 199.1705199 .1704 & 0.5 & $17: 1$ & 267.2332 & 267.2330 & 0.7 & 534.3186 & 534.3201 & 2.8 & 548.3332 & 548.3358 & 4.7 & 14.01 & $9 \Delta$ & $\omega 7$ \\
\hline 705.4719 & \multirow{2}{*}{705.4712} & 1.0 & 17.45 & \multirow{2}{*}{ PG } & $14: 0$ & 227.2018227 .2017 & 0.4 & $17: 1$ & 267.2332 & 267.2330 & 0.7 & 593.3433 & 593.346 & -4.6 & 607.3599 & 607.3617 & -3.0 & 14.02 & $9 \Delta$ & $\omega-7$ \\
\hline 705.4713 & & 0.1 & 17.75 & & 15:1 & 239.2016239 .2017 & -0.4 & $16: 0$ & 255.2330 & 255.2330 & 0.0 & 593.3425 & 593.346 & -5.9 & 607.3587 & 607.3617 & -4.9 & 14.02 & $7 \Delta$ & $\omega-7$ \\
\hline 719.4877 & \multirow[t]{2}{*}{719.4869} & 1.1 & 19.03 & \multirow[t]{2}{*}{ PG } & $16: 0$ & 255.2329255 .2330 & -0.4 & $16: 1$ & 253.2176 & 253.2173 & 1.2 & 609.3813 & 609.3773 & 6.6 & 633.3832 & 633.3773 & 9.3 & 24.00 & $9 \Delta$ & $\omega-7$ \\
\hline 719.4874 & & 0.7 & 19.52 & & 15:0 & 241.2174241 .2173 & 0.4 & $17: 1$ & 267.2331 & 267.2330 & 0.4 & 607.3596 & 607.3617 & -3.5 & 621.3796 & 621.3773 & 3.7 & 14.02 & $9 \Delta$ & $\omega-7$ \\
\hline 745.5029 & 745.5025 & 0.5 & 20.91 & PG & $17: 1$ & 267.2329267 .2330 & -0.4 & $17: 1$ & 267.2329 & 267.2330 & -0.4 & 633.3743 & 633.3773 & -4.7 & 647.3898 & 647.393 & -4.9 & 14.02 & $9 \Delta, 9 \Delta$ & $\omega-7, \omega-7$ \\
\hline 674.4789 & 674.4766 & 3.4 & 21.36 & PE & 14:0 & 227.2022227 .2017 & 2.2 & $17: 1$ & 267.2336 & 267.2330 & 2.2 & 562.3518 & 562.3514 & 0.7 & 576.3665 & 576.3671 & -1.0 & 14.01 & $9 \Delta$ & $\omega-7$ \\
\hline 733.5034 & 733.5025 & 1.2 & 21.87 & PG & $16: 0$ & 255.2332255 .2330 & 0.8 & $17: 1$ & 267.2332 & 267.2330 & 0.7 & 621.3751 & 621.3773 & -3.5 & 635.3906 & 635.393 & -3.8 & 14.02 & $9 \Delta$ & $\omega-7$ \\
\hline 688.4933 & 688.4923 & 1.5 & 24.25 & PE & 15:0 & 241.2175241 .2173 & 0.8 & $17: 1$ & 267.2332 & 267.2330 & 0.7 & 576.3668 & 576.3671 & -0.5 & 590.3782 & 590.3827 & -7.6 & 14.01 & $9 \Delta$ & $\omega-7$ \\
\hline 747.5187 & 747.5182 & 0.7 & 23.75 & PG & $16: 1$ & 253.2174253 .2173 & 0.4 & 18:0 & 283.2644 & 283.2643 & 0.4 & 637.4102 & 637.4086 & 2.5 & 661.4063 & 661.4086 & -3.5 & 00 & $9 \Delta$ & $\begin{array}{l}\omega-7 \\
\omega-7\end{array}$ \\
\hline 714.5103 & \multirow{2}{*}{714.5079} & 3.4 & 24.06 & \multirow{2}{*}{$\mathrm{PE}$} & $16: 1$ & 253.2178253 .2173 & 2.0 & $18: 1$ & 281.2493 & 281.2486 & 2.5 & 604.3991 & 604.3984 & 1.2 & 628.4047 & 628.3984 & 10.0 & 24.01 & $\begin{array}{l}9 \Delta(16: 1), \\
11 \Delta(18: 1)\end{array}$ & $\omega-7, \omega 7$ \\
\hline 714.5083 & & 0.6 & 24.54 & & $17: 1$ & 267.2329267 .2330 & -0.4 & $17: 1$ & 267.2329 & 267.2330 & -0.4 & 602.3795 & 602.3827 & -5.3 & 616.397 & 616.3984 & -2.3 & 14.02 & $9 \Delta, 9 \Delta$ & $\omega-7, \omega-7$ \\
\hline 702.5085 & 702.5079 & 0.9 & 26.70 & $\mathrm{PE}$ & 16:0 & 255.2330255 .2330 & 0.0 & $17: 1$ & 267.2331 & 267.2330 & 0.4 & 590.3792 & 590.3827 & -5.9 & 604.3984 & 604.3984 & 0.0 & 14.02 & $9 \Delta$ & $\omega-7$ \\
\hline 728.5267 & 728.5236 & 4.3 & 27.92 & $P E$ & 17:1 & 267.2337267 .2330 & 2.6 & 19:1 & 295.2647 & 295.2639 & 2.7 & $\begin{array}{l}649.4069 \\
618.4108\end{array}$ & $\begin{array}{l}649.4086 \\
618.414\end{array}$ & $\begin{array}{l}-2.6 \\
-5.2\end{array}$ & $\begin{array}{l}663.4225 \\
642.4086\end{array}$ & $\begin{array}{l}663.4243 \\
642.414\end{array}$ & $\begin{array}{l}-2.7 \\
8.4\end{array}$ & $\begin{array}{l}14.02 \\
24.00\end{array}$ & $\begin{array}{c}11 \Delta \\
9 \Delta(17: 1), \\
11 \Delta(18: 1)\end{array}$ & $\omega-7$ \\
\hline 716.5273 & & 5.2 & 29.07 & & $16: 0$ & 255.7339255 .2330 & 3.5 & $18: 1$ & 281.2496 & 281.2486 & 3.6 & 606.4194 & 606.414 & 8.9 & 630.4113 & 630.414 & -4.3 & 23.99 & 114 & $\omega-7$ \\
\hline 716.5239 & 716.5236 & 0.4 & 29.57 & $\mathrm{PE}$ & $16: 1$ & 253.2174253 .2173 & 0.4 & $18: 0$ & 283.2643 & 283.2643 & 0.0 & 606.4146 & 606.414 & 1.0 & 630.4111 & 630.414 & -4.6 & 24.00 & $9 \Delta$ & $\omega-7$ \\
\hline 716.5239 & & 0.4 & 29.89 & & $15: 0$ & 241.2174241 .2173 & 0.4 & 19:1 & 295.2643 & 295.2639 & 1.4 & 604.3932 & 604.3984 & -8.6 & 618.413 & 618.414 & -1.6 & 14.02 & $11 \Delta$ & $\omega-7$ \\
\hline 742.5415 & 742.5392 & 3.1 & 31.53 & PE & $17: 1$ & 267.233267 .2330 & 0.0 & 19:1 & 295.2643 & 295.2639 & 1.4 & 630.4122 & 630.414 & -2.9 & 644.4269 & 644.4297 & -4.3 & 14.01 & $\begin{array}{l}9 \Delta(16: 1) \\
11 \Delta(18: 1)\end{array}$ & $\omega-7, \omega-7$ \\
\hline 730.5399 & 730.5392 & 1.0 & 33.02 & $\mathrm{PE}$ & $16: 0$ & 255.2331 255.2330 & 0.4 & 19:1 & 295.2644 & 295.2639 & 1.7 & 618.4104 & 618.414 & -5.8 & 632.429 & 632.4297 & -1.1 & 14.02 & $11 \Delta$ & $\omega-7$ \\
\hline 730.5396 & & 0.5 & 33.84 & & $17: 1$ & 267.2330267 .2330 & 0.0 & 18:0 & 283.2644 & 283.2643 & 0.4 & $\begin{array}{l}618.4097 \\
646.445\end{array}$ & 618.414 & $\begin{array}{l}-7.0 \\
-0.5\end{array}$ & $\begin{array}{l}632.4281 \\
670.4503\end{array}$ & $\begin{array}{l}632.4297 \\
670.4453\end{array}$ & $\begin{array}{l}-2.5 \\
7.5\end{array}$ & $\begin{array}{l}14.02 \\
24.01\end{array}$ & $9 \Delta$ & $\omega-7$ \\
\hline 756.5578 & 756.5549 & 3.8 & 33.95 & PE & $18: 1$ & 281.2494281 .2486 & 2.8 & 19:1 & 295.2650 & 295.2639 & 3.7 & 644.4269 & 644.4297 & -4.3 & 658.4447 & 658.4453 & -0.9 & 14.02 & $11 \Delta, 11 \Delta$ & $\omega-7, \omega-7$ \\
\hline 744.5557 & 744.5549 & 1.1 & 35.58 & $\mathrm{PE}$ & $18: 1$ & 281.2489281 .2486 & 1.1 & $18: 0$ & 283.2643 & 283.2643 & 0.0 & 634.4459 & 634.4453 & 0.9 & 658.4434 & 658.4453 & -2.9 & 24.00 & $11 \Delta$ & $\omega-7$ \\
\hline 770.5709 & 770.5705 & 0.5 & 38.40 & $\mathrm{PE}$ & $19: 1$ & 295.2643295.2639 & 1.4 & $19: 1$ & 295.2643 & 295.2639 & 1.4 & 658.4431 & 658.4453 & 3.3 & 672.4579 & 672.461 & 4.6 & 14.01 & $11 \Delta, 11 \Delta$ & $\omega 7, \omega 7$ \\
\hline
\end{tabular}

Table S4. List of all identified unsaturated lipids in $K$. pneumonia lipid extract with all precursor and fragment ion ppm error values for acyl chain product ions and unsaturation element fragment ions (within 10 ppm error). Features are color coded by headgroup (PGs in red, PEs in yellow, PCs in purple), unsaturation type (cyclopropane rings in blue, double bonds in green), and unique acyl chain modifications (odd-carbon branched acyl chains in pink, hydroxyl acyl chains in brown). 


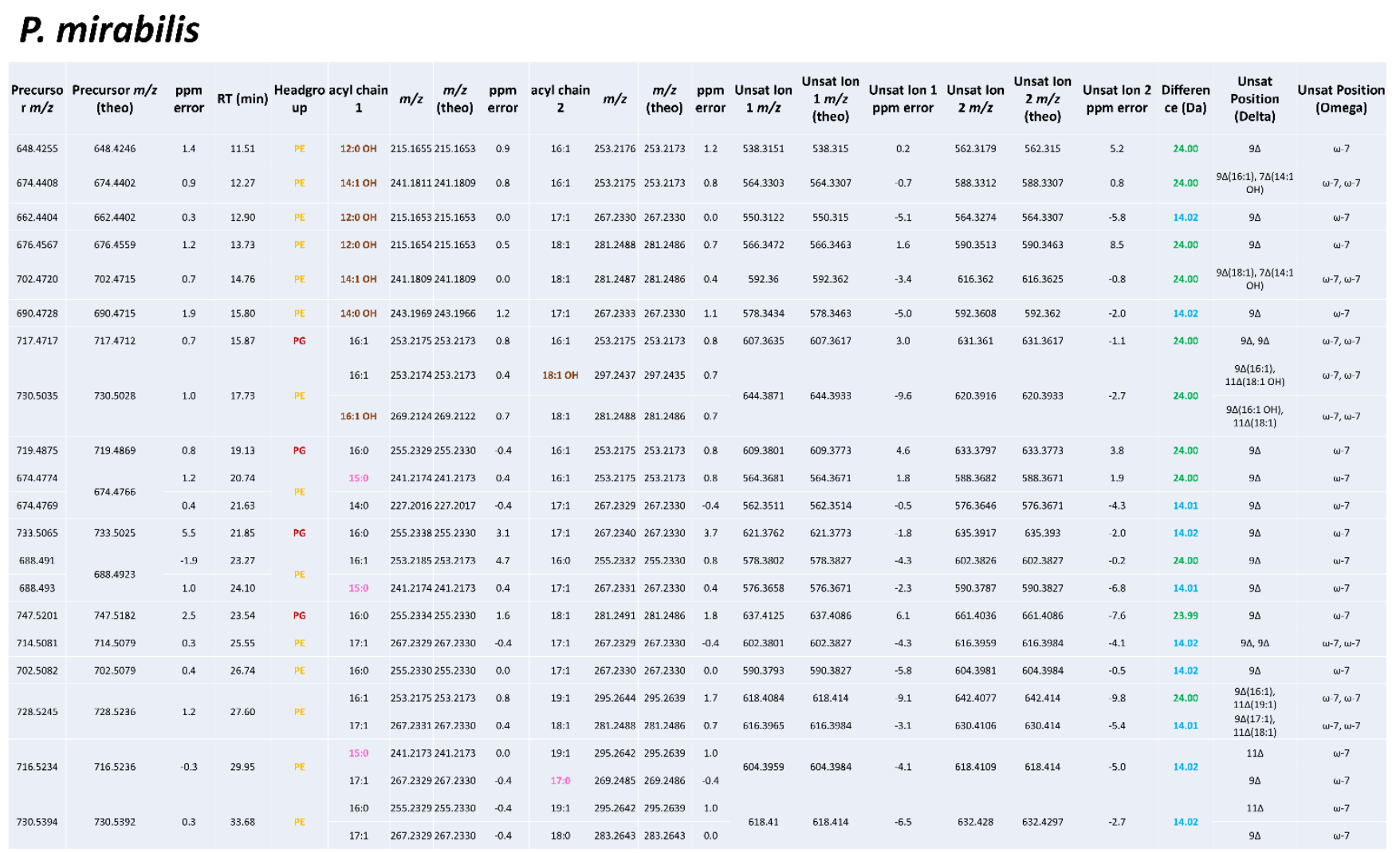

Table S5. List of all identified unsaturated lipids in P. mirabilis lipid extract with all precursor and fragment ion ppm error values for acyl chain product ions and unsaturation element fragment ions (within 10 ppm error). Features are color coded by headgroup (PGs in red, PEs in yellow, PCs in purple), unsaturation type (cyclopropane rings in blue, double bonds in green), and unique acyl chain modifications (odd-carbon branched acyl chains in pink, hydroxyl acyl chains in brown). 


\section{P. aeruginosa}

\begin{tabular}{|c|c|c|c|c|c|c|c|c|c|c|c|c|c|c|c|c|c|c|c|c|c|}
\hline $\begin{array}{l}\text { Precurso o } \\
\mathrm{r} m / z\end{array}$ & $\begin{array}{l}\begin{array}{l}\text { recursor } m / z \\
\text { (theo) }\end{array}\end{array}$ & $\begin{array}{l}\text { ppm } \\
\text { error }\end{array}$ & RT (min) ${ }^{r}$ & $\begin{array}{c}\text { Headgro } \\
\text { up }\end{array}$ & $\begin{array}{c}\text { acyl } \\
\text { chain } 1\end{array}$ & $m / \mathbf{z}$ & $\begin{array}{c}m / z \\
\text { (theo) }\end{array}$ & $\begin{array}{l}\text { ppm } \\
\text { error }\end{array}$ & $\begin{array}{c}\text { acyl } \\
\text { chain } 2\end{array}$ & $m / z$ & $\begin{array}{c}m / z \\
\text { (theo) }\end{array}$ & $\begin{array}{l}\text { ppm } \\
\text { error }\end{array}$ & $\begin{array}{l}\text { Unsat lon } \\
1 \mathrm{~m} / \mathrm{z}\end{array}$ & $\begin{array}{c}\text { Unsat lon } \\
1 \mathrm{~m} / 2 \\
\text { (theo) }\end{array}$ & $\begin{array}{l}\text { Unsat lon } 1 \\
\text { ppm error }\end{array}$ & $\begin{array}{l}\text { Unsat lon } \\
2 \mathrm{~m} / \mathrm{z}\end{array}$ & $\begin{array}{c}\text { Unsat lon } \\
2 \mathrm{~m} / 2 \\
\text { (theo) }\end{array}$ & $\begin{array}{l}\text { Unsat Ion } 2 \\
\text { ppm error }\end{array}$ & $\begin{array}{l}\text { Differen } \\
\text { ce (Da) }\end{array}$ & $\begin{array}{l}\text { Unsat } \\
\text { Position } \\
\text { (Delta) }\end{array}$ & $\begin{array}{c}\text { Unsat } \\
\text { Position } \\
\text { (Omega) }\end{array}$ \\
\hline 717.4713 & 717.4712 & 0.1 & 15.89 & PG & 16:1 & $\begin{array}{c}253.217 \\
3\end{array}$ & $\begin{array}{c}7253.217 \\
3\end{array}$ & 0.0 & $16: 1$ & 253.2173 & 3253.2173 & 0.0 & 607.3667 & 607.3617 & 8.2 & 631.3561 & 631.3617 & -8.9 & 23.99 & $9 \Delta, 9 \Delta$ & $\omega-7, \omega-7$ \\
\hline 719.4873 & 719.4869 & 0.6 & 18.61 & PG & 14:0 & $\begin{array}{c}227.201 \\
7\end{array}$ & $\begin{array}{l}1227.201 \\
7\end{array}$ & 0.0 & $18: 1$ & 281.2487 & 7281.2486 & 0.4 & 609.3801 & 609.3773 & 4.6 & 633.3816 & 633.3773 & 6.8 & 24.00 & $11 \Delta$ & $\omega-7$ \\
\hline 719.4877 & 803.4009 & 1.1 & 19.14 & ro & 16:1 & $\begin{array}{c}253.217 \\
6\end{array}$ & $\begin{array}{c}7253.217 \\
3\end{array}$ & 1.2 & 16:0 & 255.2329 & 9255.2330 & -0.4 & 609.3807 & 609.3773 & 5.6 & 633.3797 & 633.3773 & 3.8 & 24.00 & $9 \Delta$ & $\omega-7$ \\
\hline 745.5027 & 745.5025 & 0.3 & 19.67 & PG & 16:1 & $\begin{array}{c}253.217 \\
3\end{array}$ & $\begin{array}{l}7253.217 \\
3\end{array}$ & 0.0 & $18: 1$ & 281.2486 & 6281.2486 & 0.0 & 635.39 & 635.393 & -4.7 & 659.3934 & 659.393 & 0.6 & 24.00 & $\begin{array}{l}9 \Delta(16: 1), \\
11 \Delta(18: 1)\end{array}$ & $\omega-7, \omega-7$ \\
\hline 733.5023 & 733.5025 & -0.3 & 22.05 & PG & 16:0 & $\begin{array}{c}255.232 \\
9\end{array}$ & $2 \underset{0}{2255.233}$ & -0.4 & $17: 1$ & 267.2328 & 8267.2330 & -0.7 & 621.3734 & 621.3773 & 6.3 & 635.3887 & 635.393 & -6.8 & 14.02 & $9 \Delta$ & $\omega-7$ \\
\hline 747.5201 & 747.5182 & 2.5 & 23.28 & PG & $16: 0$ & 255.233 & $\begin{array}{l}355.233 \\
0\end{array}$ & 0.0 & $18: 1$ & 281.2487 & 7281.2486 & 0.4 & 637.4117 & 637.4086 & 4.9 & 661.4043 & 661.4086 & -6.5 & 23.99 & $11 \Delta$ & $\omega-7$ \\
\hline 747.5184 & & 0.3 & 23.75 & & $16: 1$ & $\begin{array}{c}253.217 \\
5\end{array}$ & $\begin{array}{c}7253.217 \\
3\end{array}$ & 0.8 & 18:0 & 283.2644 & 4283.2643 & 0.4 & 637.4125 & 637.4086 & 6.1 & 661.4031 & 661.4086 & -8.3 & 23.99 & $9 \Delta$ & $\omega-7$ \\
\hline 773.5363 & 773.5338 & 3.2 & 24.04 & PG & 18:1 & $\begin{array}{c}281.248 \\
7\end{array}$ & $\begin{array}{c}8281.248 \\
6\end{array}$ & 0.4 & $18: 1$ & 281.2487 & 7281.2486 & 0.4 & 663.4236 & 663.4243 & -1.1 & 687.4175 & 687.4243 & -9.9 & 23.99 & $11 \Delta, 11 \Delta$ & $\omega-7, \omega-7$ \\
\hline 714.5089 & 714.5079 & 1.4 & 24.77 & PE & 16:1 & $\begin{array}{c}253.217 \\
5\end{array}$ & $\begin{array}{c}7253.217 \\
3\end{array}$ & 0.8 & $18: 1$ & 281.2489 & 9281.2486 & 1.1 & 604.3975 & 604.3984 & 1.5 & 628.3958 & 628.3984 & 4.1 & 24.00 & $\begin{array}{l}9 \Delta(16: 1), \\
11 \Delta(18: 1)\end{array}$ & $\omega-7, \omega-7$ \\
\hline 702.5084 & 702.5079 & 0.7 & 25.63 & PE & 15:0 & $\begin{array}{c}241.217 \\
4\end{array}$ & $\begin{array}{l}7241.217 \\
3\end{array}$ & 0.4 & $18: 1$ & 281.2487 & 7281.2486 & 0.4 & 592.3995 & 592.3984 & 1.9 & 616.3941 & 616.3984 & -7.0 & 23.99 & $11 \Delta$ & $\omega-7$ \\
\hline 702.5087 & & 1.1 & 27.07 & & $16: 0$ & $\begin{array}{c}255.233 \\
1\end{array}$ & $\begin{array}{c}3255.233 \\
0\end{array}$ & 0.4 & $17: 1$ & 267.2332 & 2267.2330 & 0.7 & 590.3789 & 590.3827 & -6.4 & 604.3978 & 604.3984 & -1.0 & 14.02 & $9 \Delta$ & $\omega-7$ \\
\hline 761.5361 & 761.5338 & 3.0 & 27.00 & PG & 16:D & $\begin{array}{c}255.233 \\
5\end{array}$ & $\begin{array}{c}3255.233 \\
0\end{array}$ & 2.0 & 19:1 & 295.2649 & 9295.2639 & 3.4 & 649.4072 & 649.4086 & -2.2 & 663.423 & 663.4243 & -2.0 & 14.02 & $11 \Delta$ & w-7 \\
\hline 716.5258 & 716.5236 & 3.1 & 29.14 & PE & $16: 0$ & $\begin{array}{c}255.233 \\
5\end{array}$ & $3 \underset{0}{3255.233}$ & 2.0 & $18: 1$ & 281.2493 & 3281.2486 & 2.5 & 606.4199 & 606.414 & 9.7 & 630.4124 & 630.414 & -2.5 & 23.99 & $11 \Delta$ & $\omega-7$ \\
\hline 742.5399 & 742.5392 & 0.9 & 29.71 & PE & $18: 1$ & $\begin{array}{c}281.248 \\
7\end{array}$ & $\begin{array}{c}8281.248 \\
6\end{array}$ & 0.4 & $18: 1$ & 281.2487 & 7281.2486 & 0.4 & 632.4242 & 632.4297 & -8.7 & 656.4308 & 656.4297 & 1.7 & 24.01 & $11 \Delta, 11 \Delta$ & $\omega-7$ \\
\hline 742.5393 & 年 & 0.1 & 31.88 & & $17: 1$ & $\begin{array}{c}267.232 \\
9\end{array}$ & $2 \begin{array}{c}267.233 \\
0\end{array}$ & 0.4 & 19:1 & 295.2642 & 2295.2639 & 1.0 & 630.4113 & 630.414 & -4.3 & 644.4264 & 644.4297 & -5.1 & 14.02 & $\begin{array}{l}9 \Delta(17: 1), \\
11 \Delta(19: 1)\end{array}$ & $\omega-7$ \\
\hline 730.54 & 730.5392 & 1.1 & 31.13 & PE & $17: 0$ & $\begin{array}{c}269.248 \\
8\end{array}$ & $\begin{array}{c}8269.248 \\
6\end{array}$ & 0.7 & 18:1 & 281.2488 & 8281.2486 & 0.7 & 620.4337 & 620.4297 & 6.4 & 644.4255 & 644.4297 & -6.5 & 23.99 & $11 \Delta$ & $\omega-7$ \\
\hline 730.5396 & & 0.5 & 33.09 & & 16:0 & 255.233 & $3 \underset{0}{3255.233}$ & 0.0 & 19:1 & 295.2643 & 3295.2639 & 1.4 & 618.4099 & 618.414 & -6.6 & 632.4283 & 632.4297 & -2.2 & 14.02 & $11 \Delta$ & $\omega-7$ \\
\hline 818.5877 & 818.5917 & -4.9 & 31.59 & PC & $16: 0$ & $\begin{array}{c}255.232 \\
9\end{array}$ & $2 \underset{0}{2555.233}$ & -0.4 & $19 ; 1$ & 295.2642 & 2295.2639 & 1.0 & 706.4655 & 706.4665 & -1.4 & 720.4819 & 720.4821 & -0.3 & 14.02 & $11 \Delta$ & $\omega-7$ \\
\hline
\end{tabular}

Table S6. List of all identified unsaturated lipids in $P$. aeruginosa lipid extract with all precursor and fragment ion ppm error values for acyl chain product ions and unsaturation element fragment ions (within 10 ppm error). Features are color coded by headgroup (PGs in red, PEs in yellow, PCs in purple), unsaturation type (cyclopropane rings in blue, double bonds in green), and unique acyl chain modifications (odd-carbon branched acyl chains in pink, hydroxyl acyl chains in brown). 


\section{S. Typhimurium}

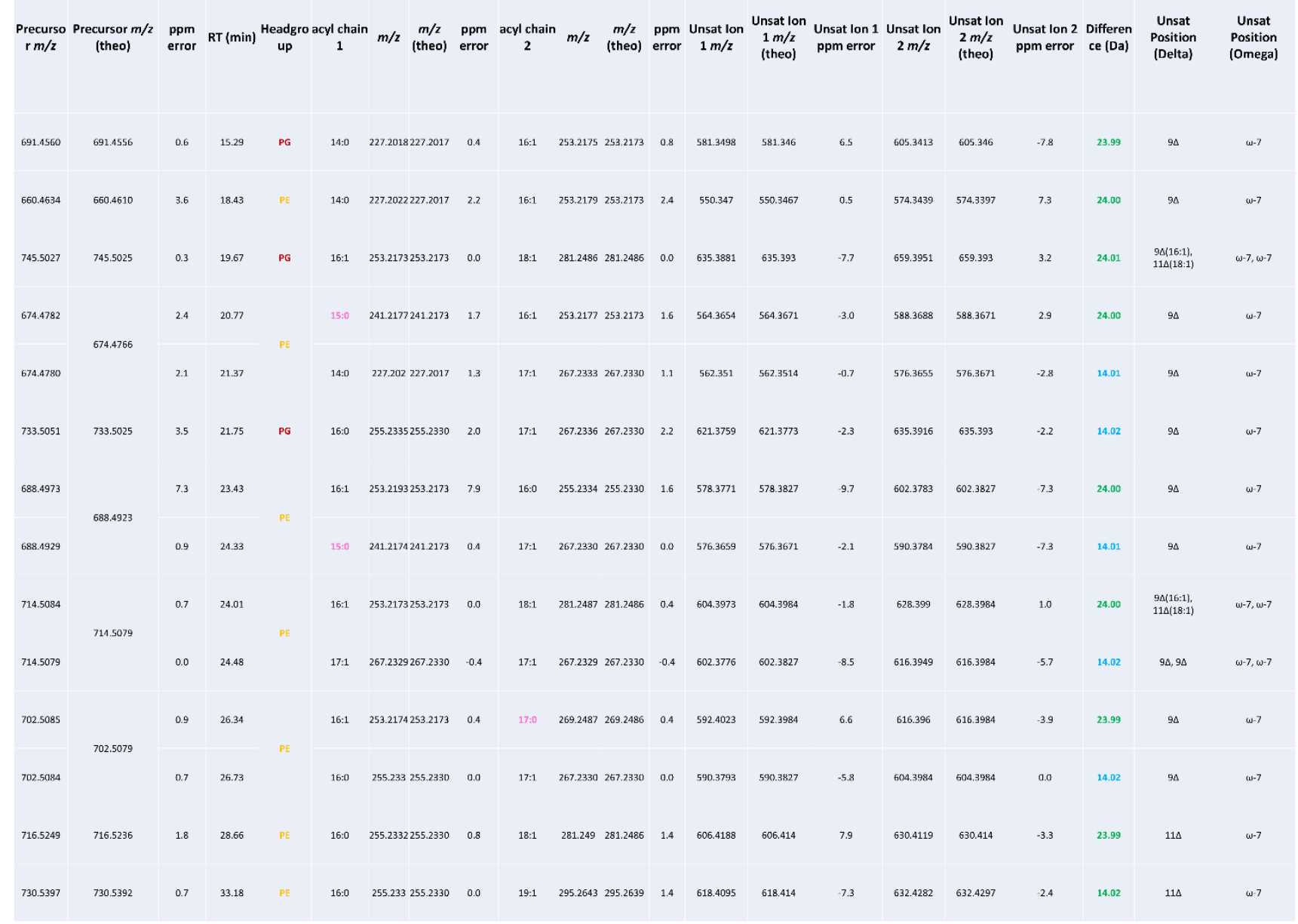

Table S7. List of all identified unsaturated lipids in S. Typhimurium lipid extract with all precursor and fragment ion ppm error values for acyl chain product ions and unsaturation element fragment ions (within 10 ppm error). Features are color coded by headgroup (PGs in red, PEs in yellow, PCs in purple), unsaturation type (cyclopropane rings in blue, double bonds in green), and unique acyl chain modifications (odd-carbon branched acyl chains in pink, hydroxyl acyl chains in brown). 


\section{V. cholerae}

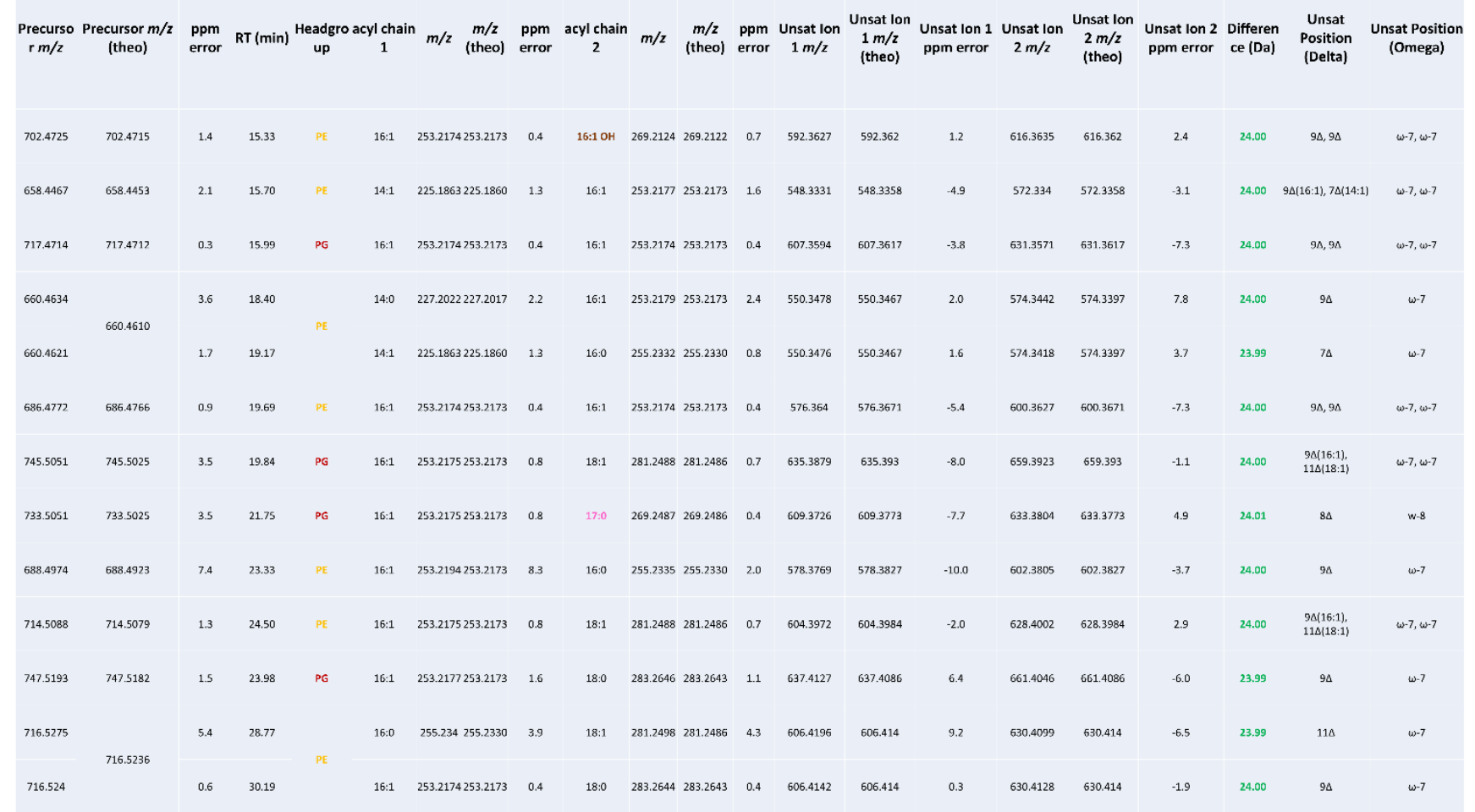

Table S8. List of all identified unsaturated lipids in V. cholera lipid extract with all precursor and fragment ion ppm error values for acyl chain product ions and unsaturation element fragment ions (within 10 ppm error). Features are color coded by headgroup (PGs in red, PEs in yellow, PCs in purple), unsaturation type (cyclopropane rings in blue, double bonds in green), and unique acyl chain modifications (odd-carbon branched acyl chains in pink, hydroxyl acyl chains in brown). 


\section{S. marcescens}

\begin{tabular}{|c|c|c|c|c|c|c|c|c|c|c|c|c|c|c|c|c|c|c|c|c|}
\hline $\begin{array}{l}\text { Precurso } \\
\mathrm{r} m / \mathbf{z}\end{array}$ & $\begin{array}{l}\text { Precursor } m / z \\
\text { (theo) }\end{array}$ & $\begin{array}{l}\text { ppm } \\
\text { error }\end{array}$ & $\mathrm{RT}(\min )$ & $\begin{array}{l}\text { Headgro } \\
\text { up }\end{array}$ & $\begin{array}{c}\text { acyl chain } \\
1\end{array}$ & $m / 2 \quad \begin{array}{c}m / z \\
\text { (theo) }\end{array}$ & $\begin{array}{l}\text { ppm } \\
\text { error }\end{array}$ & $\begin{array}{c}\text { acyl chain } \\
2\end{array}$ & $m / z$ & $\begin{array}{c}m / z \\
\text { (theo) }\end{array}$ & $\begin{array}{l}\text { ppm } \\
\text { error }\end{array}$ & $\begin{array}{c}\text { Unsat lon } \\
1 \mathrm{~m} / \mathrm{z}\end{array}$ & $\begin{array}{c}\text { Unsat lon } \\
1 \mathrm{~m} / \mathrm{z} \\
\text { (theo) }\end{array}$ & $\begin{array}{l}\text { Unsat Ion } 1 \\
\text { ppm error }\end{array}$ & $\begin{array}{l}\text { Unsat lon } \\
2 \mathrm{~m} / \mathrm{z}\end{array}$ & $\begin{array}{c}\text { Unsat lon } \\
2 \mathrm{~m} / \mathrm{z} \\
\text { (theo) }\end{array}$ & $\begin{array}{l}\text { Unsat lon } 2 \\
\text { ppm error }\end{array}$ & $\begin{array}{l}\text { Differen } \\
\text { ce (Da) }\end{array}$ & $\begin{array}{c}\text { Unsat } \\
\text { Position } \\
\text { (Delta) }\end{array}$ & $\begin{array}{l}\text { Unsat Position } \\
\text { (Omega) }\end{array}$ \\
\hline 717.4711 & 717.4712 & -0.1 & 15.82 & PG & $16: 1$ & 253.2173253 .2173 & 0.0 & 16:1 & 253.2173 & 253.2173 & 0.0 & 607.3582 & 607.3617 & -5.8 & 631.3618 & 631.3617 & 0.2 & 24.00 & $9 \Delta, 9 \Delta$ & $\omega-7, \omega-7$ \\
\hline 705.4710 & 705.4712 & -0.3 & 17.53 & PG & 14:0 & 227.2016227 .2017 & -0.4 & 17:1 & 267.2329 & 267.2330 & -0.4 & 593.342 & 593.346 & -6.7 & 607.3587 & 607.3617 & -4.9 & 14.02 & $9 \Delta$ & $\omega-7$ \\
\hline 686.4782 & 686.4766 & 2.3 & 19.16 & PE & $16: 1$ & 253.2176253 .2173 & 1.2 & 16:1 & 253.2176 & 253.2173 & 1.2 & 576.3704 & 576.3671 & 5.7 & 600.3626 & 600.3671 & -7.5 & 23.99 & $9 \Delta, 9 \Delta$ & $\omega-7, \omega-7$ \\
\hline 674.4767 & & 0.1 & 20.70 & & 15:0 & 241.2173241 .2173 & 0.0 & $16: 1$ & 253.2173 & 253.2173 & 0.0 & 564.3691 & 564.3671 & 3.5 & 588.3692 & 588.3671 & 3.6 & 24.00 & $9 \Delta$ & $\omega .7$ \\
\hline 674.4777 & & 1.6 & 21.37 & & 14:0 & 227.2019227 .2017 & 0.9 & $17: 1$ & 267.2332 & 267.2330 & 0.7 & 562.3506 & 562.3514 & -1.4 & 576.3652 & 576.3671 & -3.3 & 14.01 & $9 \Delta$ & $\omega-7$ \\
\hline 733.5068 & 733.5025 & 5.9 & 21.76 & PG & 16:0 & 255.2339255 .2330 & 3.5 & $17: 1$ & 267.2341 & 267.2330 & 4.1 & 621.3763 & 621.3773 & -1.6 & 635.3918 & 635.393 & -1.9 & 14.02 & $9 \Delta$ & $\omega-7$ \\
\hline 714.5082 & 714.5079 & 0.4 & 23.33 & PE & 17:1 & 267.233267 .2330 & 0.0 & $17: 1$ & 267.233 & 267.2330 & 0.0 & 602.3804 & 602.3827 & -3.8 & 616.3964 & 616.3984 & -3.2 & 14.02 & $9 \Delta, 9 \Delta$ & $\omega \cdot 7, \omega-7$ \\
\hline 688.4931 & 688.4923 & 1.2 & 24.18 & PE & 15:0 & 241.2175241 .2173 & 0.8 & $17: 1$ & 267.2331 & 267.2330 & 0.4 & 576.3672 & 576.3671 & 0.2 & 590.3781 & 590.3827 & -7.8 & 14.01 & $9 \Delta$ & $\omega-7$ \\
\hline 702.5086 & 702.5079 & 1.0 & 26.22 & $P E$ & 16:1 & 253.2175253 .2173 & 0.8 & $17: 0$ & 269.2487 & 269.2486 & 0.4 & 592.3994 & 592.3984 & 1.7 & 616.3947 & 616.3984 & -6.0 & 24.00 & $9 \Delta$ & $\omega-7$ \\
\hline 702.5084 & & 0.7 & 26.72 & & 16:0 & 255.233255 .2330 & 0.0 & $17: 1$ & 267.2330 & 267.2330 & 0.0 & 590.3792 & 590.3827 & -5.9 & 604.3984 & 604.3984 & 0.0 & 14.02 & $9 \Delta$ & $\omega-7$ \\
\hline 716.5248 & & 1.7 & 28.68 & & 16:0 & 255.2332255 .2330 & 0.8 & 18:1 & 281.2490 & 281.2486 & 1.4 & 606.4199 & 606.414 & 9.7 & 630.4133 & 630.414 & -1.1 & 23.99 & $11 \Delta$ & w.7. \\
\hline 716.5242 & & 0.8 & 29.31 & & $16: 1$ & 253.2175253 .2173 & 0.8 & 18:0 & 283.2644 & 283.2643 & 0.4 & 606.4144 & 606.414 & 0.7 & 630.4102 & 630.414 & -6.0 & 24.00 & $9 \Delta$ & $\omega-7$ \\
\hline 730.5394 & 730.5392 & 0.3 & 33.25 & PE & 16:0 & 255.2329255 .2330 & -0.4 & $19: 1$ & 295.2642 & 295.2639 & 1.0 & 618.4095 & 618.414 & -7.3 & 632.4277 & 632.4297 & -3.2 & 14.02 & $11 \Delta$ & $\omega-7$ \\
\hline 730.5392 & & 0.0 & 33.48 & & 17:1 & 267.2329267 .2330 & 0.4 & 18:0 & 283.2642 & 283.2643 & 0.4 & 618.4093 & 618.414 & 7.6 & 632.4289 & 632.4297 & -1.3 & 14.02 & $9 \Delta$ & $\omega .7$ \\
\hline
\end{tabular}

Table S9. List of all identified unsaturated lipids in S. marcescens lipid extract with all precursor and fragment ion ppm error values for acyl chain product ions and unsaturation element fragment ions (within 10 ppm error). Features are color coded by headgroup (PGs in red, PEs in yellow, PCs in purple), unsaturation type (cyclopropane rings in blue, double bonds in green), and unique acyl chain modifications (odd-carbon branched acyl chains in pink, hydroxyl acyl chains in brown). 


\section{C. jejuni}

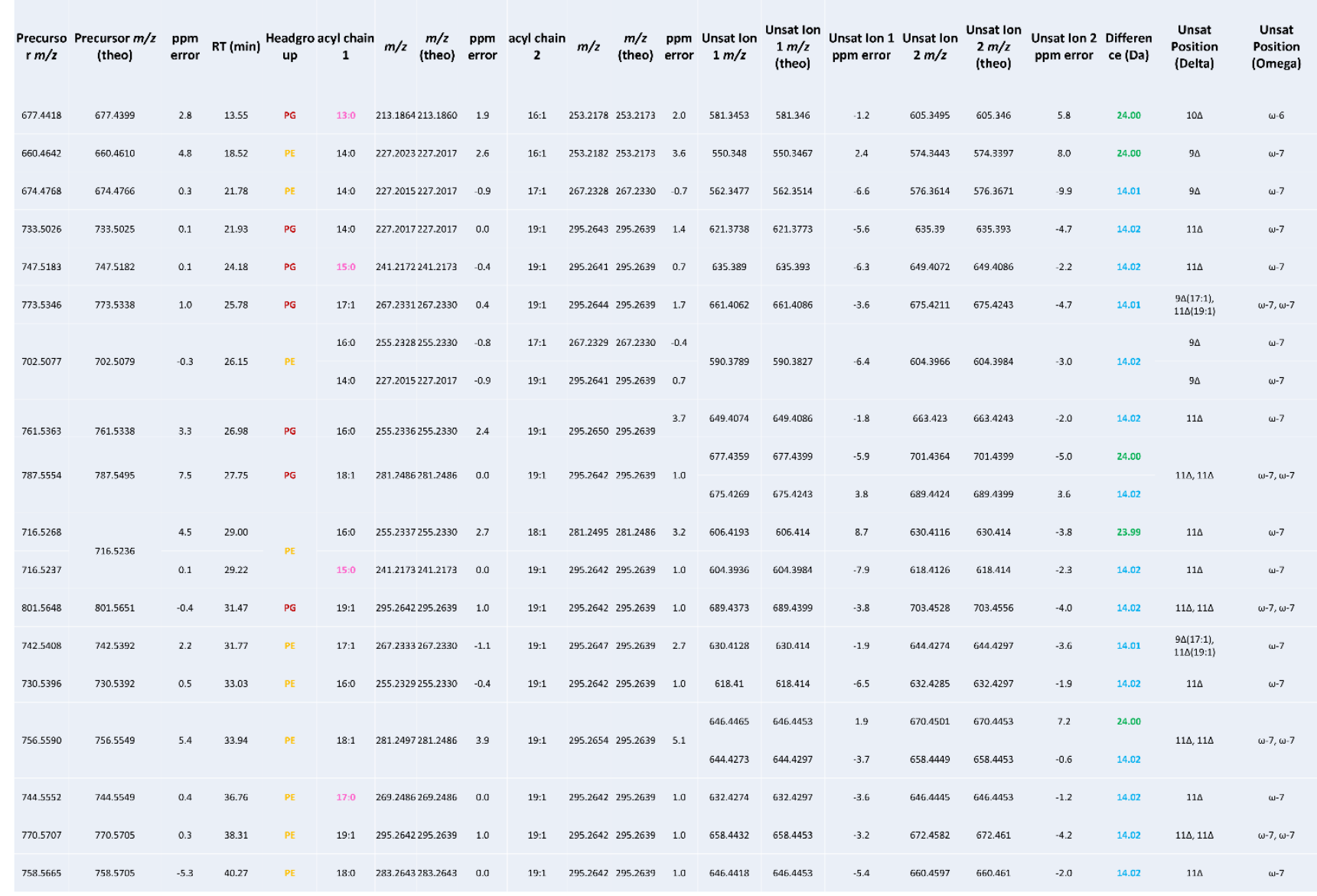

Table S10. List of all identified unsaturated lipids in C. jejuni lipid extract with all precursor and fragment ion ppm error values for acyl chain product ions and unsaturation element fragment ions (within 10 ppm error). Features are color coded by headgroup (PGs in red, PEs in yellow, PCs in purple), unsaturation type (cyclopropane rings in blue, double bonds in green), and unique acyl chain modifications (odd-carbon branched acyl chains in pink, hydroxyl acyl chains in brown). 


\section{Y. pseudotuberculosis}

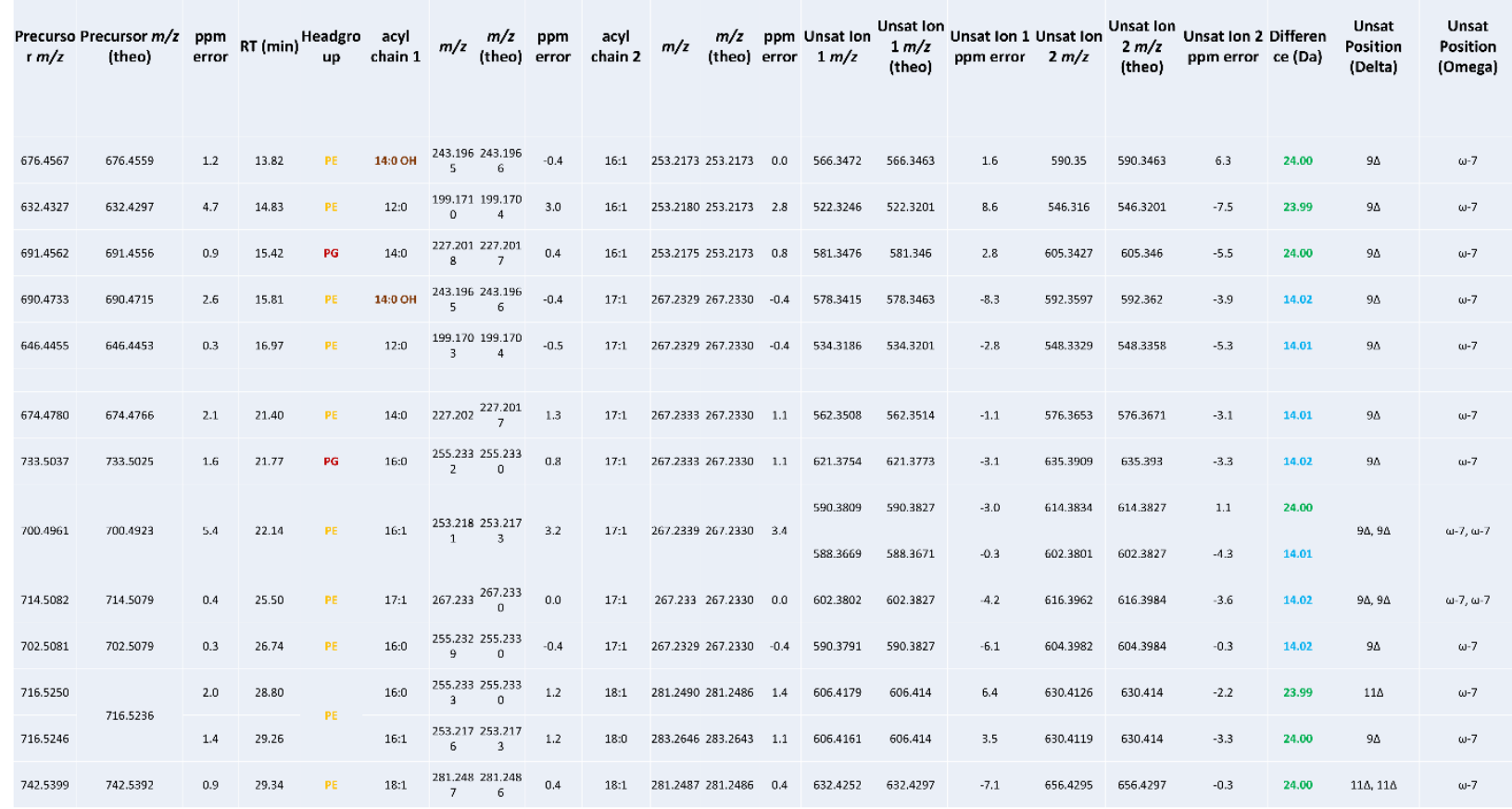

Table S11. List of all identified unsaturated lipids in Y. pseudotuberculosis lipid extract with all precursor and fragment ion ppm error values for acyl chain product ions and unsaturation element fragment ions (within 10 ppm error). Features are color coded by headgroup (PGs in red, PEs in yellow, PCs in purple), unsaturation type (cyclopropane rings in blue, double bonds in green), and unique acyl chain modifications (odd-carbon branched acyl chains in pink, hydroxyl acyl chains in brown). 


\section{B. subtilis}

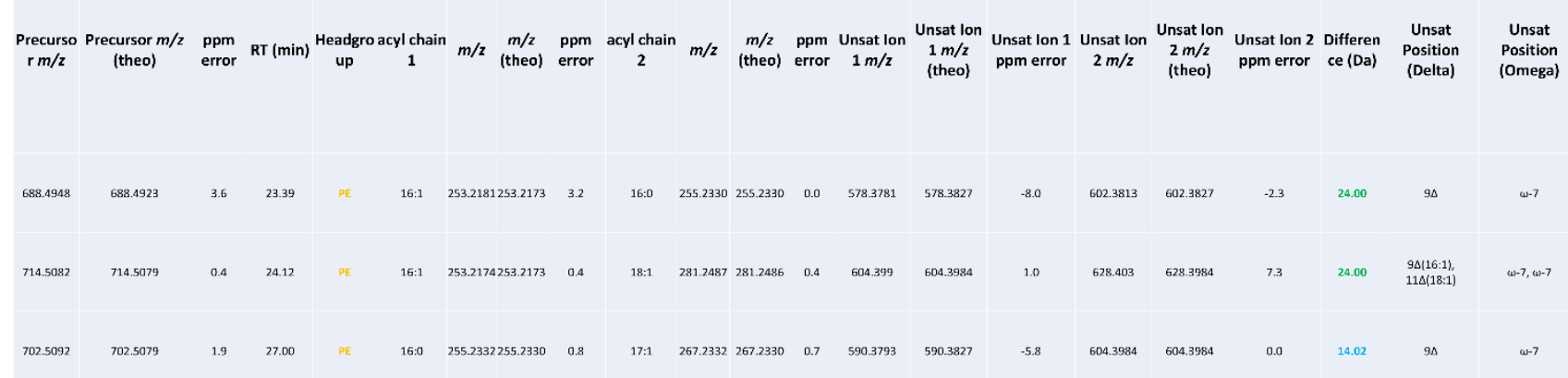

Table S12. List of all identified unsaturated lipids in B. subtilis lipid extract with all precursor and fragment ion ppm error values for acyl chain product ions and unsaturation element fragment ions (within 10 ppm error). Features are color coded by headgroup (PGs in red, PEs in yellow, PCs in purple), unsaturation type (cyclopropane rings in blue, double bonds in green), and unique acyl chain modifications (odd-carbon branched acyl chains in pink, hydroxyl acyl chains in brown). 


\section{L. plantarum}

\begin{tabular}{|c|c|c|c|c|c|c|c|c|c|c|c|c|c|c|c|c|c|c|c|c|c|c|}
\hline $\begin{array}{c}\text { Precurso } \\
\mathrm{r} m / \mathbf{z}\end{array}$ & $\begin{array}{l}\text { Precursor } m / z \\
\text { (theo) }\end{array}$ & $\begin{array}{l}\text { ppm } \\
\text { error }\end{array}$ & $\mathrm{RT}(\mathrm{min})$ & $\begin{array}{l}\text { Headgro } \\
\text { up }\end{array}$ & $\begin{array}{c}\text { acyl } \\
\text { chain } 1\end{array}$ & $m / z$ & $\begin{array}{c}m / z \\
\text { (theo) }\end{array}$ & $\begin{array}{l}\text { ppm } \\
\text { error }\end{array}$ & $\begin{array}{c}\text { acyl } \\
\text { chain } 2\end{array}$ & $m / z$ & $\begin{array}{c}m / z \\
\text { (theo) }\end{array}$ & $\begin{array}{l}\text { ppm } \\
\text { error }\end{array}$ & $\begin{array}{c}\text { Unsat } \\
\text { Ion } 1 \\
m / z\end{array}$ & $\begin{array}{l}\text { Unsat lon } \\
1 \mathrm{~m} / \mathrm{z} \\
\text { (theo) }\end{array}$ & $\begin{array}{l}\text { Unsat lon } \\
1 \text { ppm } \\
\text { error }\end{array}$ & $\begin{array}{l}\text { Unsat lon } 2 \\
\mathrm{~m} / \mathrm{z}\end{array}$ & $\begin{array}{l}\text { Unsat lon } \\
2 \mathrm{~m} / \mathrm{z} \\
\text { (theo) }\end{array}$ & $\begin{array}{l}\text { Unsat lon } \\
2 \text { ppm } \\
\text { error }\end{array}$ & $\begin{array}{l}\text { Difference } \\
\text { (Da) }\end{array}$ & $\begin{array}{l}\text { Unsat } \\
\text { Position } \\
\text { (Delta) }\end{array}$ & $\begin{array}{c}\text { Unsat } \\
\text { Position } \\
\text { (Omega) }\end{array}$ & $\begin{array}{c}\text { Precursor } \\
\mathrm{m} / \mathrm{z}\end{array}$ \\
\hline 717.4712 & 717.4712 & 0.0 & 1823 & 15.85 & PG & 16:1 & $\begin{array}{c}253.217 \\
3\end{array}$ & $\begin{array}{c}253.217 \\
3\end{array}$ & 0.0 & $16: 1$ & 253.2173 & 253.2173 & 0.0 & 607.3586 & 607.3617 & -5.1 & 631.3595 & 631.3617 & -3.5 & 24.00 & $9 \Delta$ & $\omega-7$ \\
\hline 719.4888 & \multirow{2}{*}{719.4869} & 2.6 & 2015 & 18.80 & \multirow{2}{*}{ PG } & 14:0 & $\begin{array}{c}227.202 \\
1\end{array}$ & $\begin{array}{c}227.201 \\
7\end{array}$ & 1.8 & 18:1 & 281.2492 & 281.2486 & 2.1 & 609.3845 & 609.3773 & 11.8 & 633.3824 & 633.3773 & 8.1 & 24.00 & $11 \Delta$ & $\omega-7$ \\
\hline 719.4879 & & 1.4 & 2024 & 19.03 & & $16: 1$ & $\begin{array}{c}253.217 \\
7\end{array}$ & $\begin{array}{c}253.217 \\
3\end{array}$ & 1.6 & $16: 0$ & 255.233 & 255.2330 & 0.0 & 609.3815 & 609.3773 & 6.9 & 633.3845 & 633.3773 & 11.4 & 24.00 & $9 \Delta$ & $\omega-7$ \\
\hline 745.5040 & 745.5025 & 2.0 & 2067 & 20.23 & PG & 16:1 & $\begin{array}{c}253.217 \\
9\end{array}$ & $\begin{array}{c}253.217 \\
3\end{array}$ & 2.4 & 18:1 & 281.2491 & 281.2486 & 1.8 & 635.3878 & 635.393 & -8.2 & 659.3986 & 659.393 & 8.5 & 24.01 & $\begin{array}{l}9 \Delta(16: 1), \\
11 \Delta(18: 1)\end{array}$ & $\omega-7, \omega-7$ \\
\hline 733.5024 & 733.5025 & -0.1 & 2128 & 21.70 & PG & 14:0 & $\begin{array}{c}227.201 \\
6\end{array}$ & $\begin{array}{c}227.201 \\
7\end{array}$ & -0.4 & 19:1 & \multicolumn{2}{|c|}{$295.2642 \quad 295.2639$} & 1.0 & $\begin{array}{l}621.3737 \\
593.3429\end{array}$ & 621.3773 & -5.8 & 635.3885 & 635.393 & -7.1 & 14.01 & $11 \Delta$ & $\omega-7$ \\
\hline \multirow{2}{*}{747.5201} & \multirow{2}{*}{747.5182} & \multirow{2}{*}{2.5} & \multirow[t]{2}{*}{2227} & \multirow{2}{*}{24.59} & \multirow[t]{2}{*}{$P G$} & 16:1 & $\underset{4}{253.217}$ & $\begin{array}{c}253.217 \\
3\end{array}$ & 0.4 & 18:0 & 283.2643 & 283.2643 & 0.0 & \multirow{2}{*}{637.4096} & \multirow{2}{*}{637.4086} & \multirow{2}{*}{1.6} & \multirow{2}{*}{661.4095} & \multirow{2}{*}{661.4086} & \multirow[t]{2}{*}{1.4} & \multirow{2}{*}{24.00} & $9 \Delta$ & $\omega-7$ \\
\hline & & & & & & $16: 0$ & $\begin{array}{c}255.232 \\
9\end{array}$ & $\begin{array}{l}255.233 \\
0\end{array}$ & -0.4 & 18:1 & 281.2487 & 281.2486 & 0.4 & & & & & & & & $11 \Delta$ & $\omega-7$ \\
\hline \multirow[t]{2}{*}{761.5354} & \multirow[t]{2}{*}{761.5338} & \multirow[t]{2}{*}{2.1} & \multirow[t]{2}{*}{2333} & \multirow[t]{2}{*}{27.45} & \multirow[t]{2}{*}{ PG } & $16: 0$ & $\begin{array}{l}255.233 \\
4\end{array}$ & $\begin{array}{l}255.233 \\
0\end{array}$ & 1.6 & 19:1 & 295.2647 & 295.2639 & 2.7 & 649.4067 & 649.4086 & -2.9 & 663.4225 & 663.4243 & -2.7 & 14.02 & $11 \Delta$ & $\omega-7$ \\
\hline & & & & & & & & & & & & & & 621.3784 & 621.3773 & 1.8 & 635.3928 & 635.393 & -0.3 & 14.01 & $9 \Delta$ & $\omega-9$ \\
\hline 775.5500 & 775.5495 & 0.6 & 2396 & 29.02 & PG & $18: 1$ & $\begin{array}{c}281.248 \\
9\end{array}$ & $\begin{array}{c}281.248 \\
6\end{array}$ & 1.1 & 18:0 & 283.2643 & 283.2643 & 0.0 & 665.4409 & 665.4399 & 1.5 & 689.4399 & 689.4399 & 0.0 & 24.00 & $11 \Delta$ & $\omega-7$ \\
\hline
\end{tabular}

Table S13. List of all identified unsaturated lipids in L. plantarum lipid extract with all precursor and fragment ion ppm error values for acyl chain product ions and unsaturation element fragment ions (within 10 ppm error). Features are color coded by headgroup (PGs in red, PEs in yellow, PCs in purple), unsaturation type (cyclopropane rings in blue, double bonds in green), and unique acyl chain modifications (odd-carbon branched acyl chains in pink, hydroxyl acyl chains in brown). 


\section{Polyunsaturated fatty acids within GPLs}

\begin{tabular}{|c|c|c|c|c|}
\hline Acyl Chain & $m / z_{\text {theoretical }}$ & $m / z_{\text {observed }}$ & error (ppm) & Sample \\
\hline \multirow[b]{2}{*}{$11: 2 \mathrm{OH}$} & \multirow[b]{2}{*}{197.1183} & 197.1183 & 0.00 & Escherichia coli \\
\hline & & 197.1183 & 0.00 & Salmonella enterica Typhimurium \\
\hline \multirow{2}{*}{$16: 4$} & \multirow{2}{*}{247.1704} & 247.1705 & 0.40 & Klebsiella pneumoniae \\
\hline & & 247.1705 & 0.40 & Bacillus subtilis \\
\hline \multirow{6}{*}{$16: 2$} & \multirow{6}{*}{251.2017} & 251.2016 & -0.40 & Klebsiella pneumoniae \\
\hline & & 251.2019 & 0.80 & Proteus mirabilis \\
\hline & & 251.2017 & 0.00 & Vibrio cholerae \\
\hline & & 251.2017 & 0.00 & Lactobacillus plantarum \\
\hline & & 251.2013 & -1.59 & Escherichia coli \\
\hline & & 251.2016 & -0.40 & Pseudomonas aeruginosa \\
\hline \multirow{2}{*}{$16: 3 \mathrm{OH}$} & \multirow{2}{*}{265.1809} & 265.1810 & 0.38 & Vibrio cholerae \\
\hline & & 265.1810 & 0.38 & Escherichia coli \\
\hline $17: 2$ & 265.2173 & 265.2173 & 0.00 & Klebsiella pneumoniae \\
\hline \multirow{2}{*}{$16: 2 \mathrm{OH}$} & \multirow{2}{*}{267.1967} & 267.1966 & 0.37 & Escherichia coli \\
\hline & & 267.1966 & 0.37 & Enterobacter cloacae \\
\hline \multirow{2}{*}{$18: 4$} & \multirow{2}{*}{275.2017} & 275.2017 & 0.00 & Klebsiella pneumoniae \\
\hline & & 275.2018 & 0.36 & Bacillus subtilis \\
\hline $18: 3$ & 277.2173 & 277.2171 & -0.72 & Campylobacter jejuni \\
\hline \multirow{8}{*}{$18: 2$} & \multirow{8}{*}{279.2330} & 279.2329 & -0.36 & Klebsiella pneumoniae \\
\hline & & 279.2328 & -0.72 & Proteus mirabilis \\
\hline & & 279.233 & 0.00 & Vibrio cholerae \\
\hline & & 279.2328 & -0.72 & Campylobacter jejuni \\
\hline & & 279.2329 & -0.36 & Escherichia coli \\
\hline & & 279.2331 & 0.36 & Lactobacillus plantarum \\
\hline & & 279.2327 & -1.07 & Enterobacter cloacae \\
\hline & & 279.2330 & 0.00 & Pseudomonas aeruginosa \\
\hline $19: 2$ & 293.2486 & 293.2486 & 0.00 & Klebsiella pneumoniae \\
\hline \multirow{3}{*}{$18: 2 \mathrm{OH}$} & \multirow{3}{*}{295.2279} & 295.2281 & 0.68 & Vibrio cholerae \\
\hline & & 295.2277 & -0.68 & Escherichia coli \\
\hline & & 295.2277 & -0.68 & Yersinia pseudotuberculosis \\
\hline $20: 4$ & 303.2330 & 303.2330 & 0.00 & Klebsiella pneumoniae \\
\hline
\end{tabular}

Table S14. List of identified polyunsaturated fatty acids (PUFAs) within GPLs with observed and theoretical acyl chain product ion $\mathrm{m} / \mathrm{z}$ values along with the bacterial lipid extract they were identified in. 


\section{Hydroxyl fatty acids within GPLs}

\begin{tabular}{|c|c|c|c|c|}
\hline Acyl Chai & $/ / Z_{\text {theoreti }}$ & $n / z_{\text {observe }}$ & or (ppm) & Sample \\
\hline $8: 1 \mathrm{OH}$ & 157.0870 & 157.0870 & 0.00 & Escherichia coli \\
\hline & & 171.1027 & 0.00 & Escherichia coli \\
\hline & & 171.1027 & 0.00 & Enterobacter cloacae \\
\hline & & 171.1027 & 0.00 & Klebsiella pneumoniae \\
\hline $0.1 \Omega \mathrm{H}$ & 1711970 & 171.1027 & 0.00 & Proteus mirabilis \\
\hline Y:1 UH & $1 / 1.1027$ & 171.1028 & -0.58 & Salmonella enterica Typhimurium \\
\hline & & 171.1027 & 0.00 & Vibrio cholerae \\
\hline & & 171.1026 & 0.58 & Serratia marcescens \\
\hline & & 171.1026 & 0.58 & Yersinia pseudotuberculosis \\
\hline & & 187.1339 & 0.53 & Escherichia coli \\
\hline بـ & 1071310 & 187.1340 & 0.00 & Proteus mirabilis \\
\hline 10:0 OH & $18 \% .1340$ & 187.1340 & 0.00 & Salmonella enterica Typhimurium \\
\hline & & 187.1340 & 0.00 & Yersinia pseudotuberculosis \\
\hline & & 197.1183 & 0.00 & Escherichia coli \\
\hline $11: 2 \mathrm{OH}$ & 197.1183 & 197.1183 & 0.00 & Salmonella enterica Typhimurium \\
\hline $11: 1 \mathrm{OH}$ & 199.1340 & 199.1339 & 0.50 & Escherichia coli \\
\hline $12: 1 \mathrm{OH}$ & 213.1496 & 213.1496 & 0.00 & Proteus mirabilis \\
\hline & & 215.1653 & 0.00 & Escherichia coli \\
\hline $12: 0 \mathrm{OH}$ & 215.1653 & 215.1655 & -0.93 & Proteus mirabilis \\
\hline & & 215.1654 & -0.46 & Salmonella enterica Typhimurium \\
\hline $14.1 \mathrm{OH}^{-2}-\mathrm{r}$ & & 241.1811 & -0.83 & Proteus mirabilis \\
\hline 14:1 OH & 241.1809 & 241.1811 & -0.83 & Vibrio cholerae \\
\hline & & 243.1966 & 0.00 & Escherichia coli \\
\hline & & 243.1966 & 0.00 & Proteus mirabilis \\
\hline $14: 0 \mathrm{OH}$ & 243.1966 & 243.1967 & -0.41 & Salmonella enterica Typhimurium \\
\hline & & 243.1969 & -1.23 & Vibrio cholerae \\
\hline & & 243.1965 & 0.41 & Yersinia pseudotuberculosis \\
\hline $16.3 \mathrm{OH}^{-1}$ & & 265.1810 & -0.38 & Vibrio cholerae \\
\hline $16: 3 \mathrm{UH}$ & 265.1809 & 265.1810 & -0.38 & Escherichia coli \\
\hline & & 267.1967 & -0.37 & Escherichia coli \\
\hline & & 267.1966 & 0.00 & Enterobacter cloacae \\
\hline & & 267.1965 & 0.37 & Salmonella enterica Typhimurium \\
\hline $16: 2 \mathrm{OH}$ & 267.1966 & 267.1967 & -0.37 & Vibrio cholerae \\
\hline & & 267.1967 & -0.37 & Serratia marcescens \\
\hline & & 267.1966 & 0.00 & Yersinia pseudotuberculosis \\
\hline & & 269.2124 & -0.74 & Escherichia coli \\
\hline & & 269.2121 & 0.37 & Klebsiella pneumoniae \\
\hline & & 269.2123 & -0.37 & Proteus mirabilis \\
\hline & & 269.2123 & -0.37 & Pseudomonas aeruginosa \\
\hline $16: 1 \mathrm{OH}$ & 269.2122 & 269.2121 & 0.37 & Salmonella enterica Typhimurium \\
\hline & & 269.2124 & -0.74 & Vibrio cholerae \\
\hline & & 269.2122 & 0.00 & Serratia marcescens \\
\hline & & 269.2120 & 0.74 & Yersinia pseudotuberculosis \\
\hline & & 271.2281 & -0.74 & Proteus mirabilis \\
\hline $16: 0 \mathrm{OH}$ & 271.2279 & 271.2277 & 0.74 & Campylobacter jejuni \\
\hline & & 271.2278 & 0.37 & Yersinia pseudotuberculosis \\
\hline & & 295.2281 & -0.68 & Vibrio cholerae \\
\hline $18: 2 \mathrm{OH}$ & 295.2279 & 295.2277 & 0.68 & Escherichia coli \\
\hline & & 295.2277 & 0.68 & Yersinia pseudotuberculosis \\
\hline & & 297.2437 & -0.67 & Proteus mirabilis \\
\hline $18: 1 \mathrm{OH}$ & 297.2435 & 297.2436 & -0.34 & Pseudomonas aeruginosa \\
\hline $18: 0 \mathrm{OH}$ & 299.2592 & 299.2590 & 0.67 & Vibrio cholerae \\
\hline
\end{tabular}

Table S15. List of identified hydroxyl acyl chains within GPLs with observed and theoretical acyl chain product ion $\mathrm{m} / \mathrm{z}$ values along with the bacterial lipid extract they were identified in. 


\section{Identifying hydroxyl fatty acids - isobaric acyl chains}

$\begin{array}{cccccc}\begin{array}{c}\text { OH acyl } \\ \text { chain }\end{array} & m / z & \begin{array}{c}\text { Unmodified acyl } \\ \text { chain }\end{array} & m / z & \text { Difference (Da) } & \begin{array}{c}\text { Difference } \\ \text { (ppm) }\end{array} \\ \text { 8:1 OH } & 157.0870 & 9: 0 & 157.1234 & 0.036 & 231.7 \\ 9: 1 \mathrm{OH} & 171.1027 & 10: 0 & 171.1391 & 0.036 & 212.7 \\ 11: 2 \mathrm{OH} & 197.1183 & 12: 1 & 197.1547 & 0.036 & 184.6 \\ 11: 1 \mathrm{OH} & 199.1340 & 12: 0 & 199.1704 & 0.036 & 182.8 \\ 12: 1 \mathrm{OH} & 213.1496 & 13: 0 & 213.186 & 0.036 & 170.8 \\ 14: 1 \mathrm{OH} & 241.1809 & 15: 0 & 241.2173 & 0.036 & 150.9 \\ 16: 3 \mathrm{OH} & 265.1809 & 17: 2 & 265.2173 & 0.036 & 137.3 \\ 16: 2 \mathrm{OH} & 267.1966 & 17: 1 & 267.233 & 0.036 & 136.2 \\ 16: 1 \mathrm{OH} & 269.2122 & 17: 0 & 269.2486 & 0.036 & 135.2 \\ 18: 2 \mathrm{OH} & 295.2279 & 19: 1 & 295.2643 & 0.036 & 123.3 \\ 18: 1 \mathrm{OH} & 297.2435 & 19: 0 & 297.2799 & 0.036 & 122.5\end{array}$

Table S16. List of identified $\mathrm{OH}$ acyl chains with their respective acyl chain product ion $\mathrm{m} / \mathrm{z}$ values along with unmodified (non-hydroxyl) acyl chains which are isobaric with each hydroxyl acyl chain. The small difference in mass between hydroxyl acyl chains and unmodified acyl chains showcases the need for high-resolution high-mass accuracy instrumentation for identification of hydroxyl acyl chains. 


\title{
Lysyl-PGs in Gram-negative and Gram-positive bacteria
}

\author{
Bacterium \\ Precursor $m / z$ (exp) \\ ID \\ 779.5192 \\ Lysyl PG 13:0_14:0 \\ 793.535 \\ Lysyl PG 13:0_15:0 \\ Campylobacter jejuni \\ 807.5516 \\ Lysyl PG 13:0_16:0 \\ 821.5665 \\ Lysyl PG 13:0_17:0 \\ 835.5818 \\ Lysyl PG 15:0_16:0 \\ Bacillus subtilis \\ 821.5665 \\ Lysyl PG 15:0/15:0 \\ 821.5659 \\ Lysyl PG 15:0/15:0 \\ 875.6149 \\ Lysyl PG 16:0_18:1 \\ Lactobacillus plantarum \\ 889.6292 \\ Lysyl PG 16:0_19:1 \\ 877.6284 \\ Lysyl PG 16:0_18:0
}

Table S17. List of identified lysyl-PGs with their respective precursor $m / z$ values in Gram-negative $C$. jejuni and Gram-positive B. subtilis and L. plantarum. 